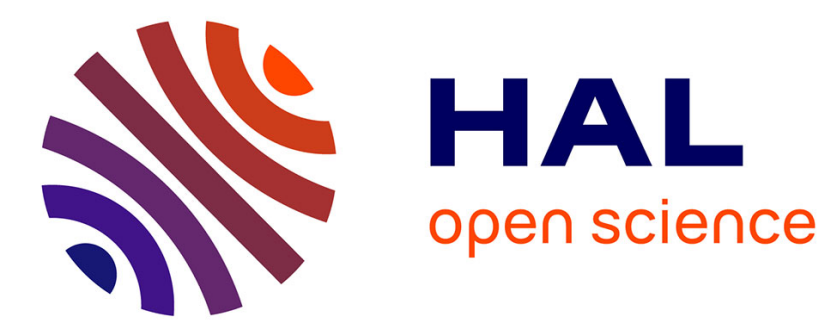

\title{
On the convergence of linear switched systems
}

Ulysse Serres, Jean-Claude Vivalda, Pierre Riedinger

\section{To cite this version:}

Ulysse Serres, Jean-Claude Vivalda, Pierre Riedinger. On the convergence of linear switched systems. IEEE Transactions on Automatic Control, 2011, 56 (2), pp.320 - 332. 10.1109/TAC.2010.2054950 . hal-00371104v1

\section{HAL Id: hal-00371104 https://hal.science/hal-00371104v1}

Submitted on 26 Mar 2009 (v1), last revised 5 Jun 2012 (v2)

HAL is a multi-disciplinary open access archive for the deposit and dissemination of scientific research documents, whether they are published or not. The documents may come from teaching and research institutions in France or abroad, or from public or private research centers.
L'archive ouverte pluridisciplinaire HAL, est destinée au dépôt et à la diffusion de documents scientifiques de niveau recherche, publiés ou non, émanant des établissements d'enseignement et de recherche français ou étrangers, des laboratoires publics ou privés. 


\title{
On the convergence of linear switched systems
}

\author{
Ulysse Serres * $^{*} \quad$ Jean-Claude Vivalda ${ }^{\dagger} \quad$ Pierre Riedinger ${ }^{\ddagger}$
}

March 26, 2009

\begin{abstract}
This paper investigates sufficient conditions for the convergence to zero of the trajectories of linear switched systems. We provide a collection of results that use weak dwell-time, dwell-time, strong dwell-time, permanent and persistent excitation hypothesis. The obtained results are shown to be tight by counterexample. Finally, we apply our result to the three-cell converter.
\end{abstract}

Keywords: Switched systems, dwell-time, stability, omega-limit set, three-cell converter.

\section{Introduction}

\subsection{Background}

Switched systems have attracted a growing interest in recent years $[11,17]$. Such systems are common across a diverse range of application areas. As an example, switched systems modeling plays a major role in the field of power systems where interactions between continuous dynamics and discrete events are an intrinsic part of power system dynamic behavior.

In the study of stability of equilibrium points of differential systems, specific results for switched and hybrid systems have been developed: see $[6,10]$ for multiple Lyapunov based approach, [18] for Lie Algebra based results, $[9,16]$ for an approach based on dynamical systems techniques, and [24] for a survey of stability criteria for switched and hybrid systems. In the context of switched systems, recent investigations (see $[2,8,13,19,20]$ ) provide interesting contributions leading to extremely general results that require little structure on the family of solutions of the hybrid system $([12,23])$.

Typically, linear switched systems are represented by equations of form

$$
\dot{x}(t)=A_{\sigma(t)} x(t), \quad x \in \mathbb{R}^{d}, \quad t \in \mathbb{R}_{+},
$$

where $\sigma(\cdot)$ denotes a piecewise constant signal that effectively switches the right-hand-side of the differential equation by selecting different matrices from a finite family $\mathscr{F}$.

In the present paper we aim to find some tight sufficient conditions on the signal $t \mapsto \sigma(t)$ in order to insure the convergence of the switched system to the origin. Our aim leads us to define several new notions of dwell-time that differ somewhat from the ones introduced

\footnotetext{
*Institut Élie Cartan de Nancy - UMR 7502 - Nancy-Université - CNRS - INRIA - BP 239 - F-54506 Vandœuvre-lès-Nancy Cedex - France - email: ulysse.serres@iecn.u-nancy.fr

${ }^{\dagger}$ Inria Nancy Grand Est \& LMAM (UMR CNRS 7122) - Université de Metz - Ile du Saulcy - F-57 045 Metz Cedex 01 - France - email: vivalda@loria.fr

${ }^{\ddagger}$ Cran - UMR 7039 - Ensem - 2, av. de la Forêt de Haye - F-56 516 Vandœuvre-lès-Nancy Cedex- France) - email: pierre.riedinger@ensem.inpl-nancy.fr
} 
in $[12,13,23]$. We are also led to define the notion of persistent excitation which ensures the convergence of the solutions of the system to a minimal invariant set $M$.

We discuss the asymptotic properties of a switched linear systems whose matrices are only assumed to be stable (not necessarily asymptotically stable). More precisely, a finite family of squared matrices of the same size $d, \mathscr{F}=\left\{A_{1}, \ldots, A_{N}\right\}$ is considered; we assume that there exists a positive definite matrix $P$ such that for every $A_{i}$ in $\mathscr{F}$,

$$
x^{\mathrm{T}}\left(A_{i}^{\mathrm{T}} P+P A_{i}\right) x \leqslant 0, \quad x \in \mathbb{R}^{d} .
$$

We reformulate the switched linear system (1.1) as an affine control system

$$
\dot{x}(t)=\sum_{i=1}^{N} \alpha_{i}(t) A_{i} x(t), \quad x(0)=x_{0},
$$

where $x(t) \in \mathbb{R}^{d}, \alpha_{i}(t) \in\{0,1\}$ and $\sum_{i=1}^{N} \alpha_{i}(t)=1$. The class of switching signals considered in this work is not equal to the whole $\boldsymbol{L}^{\infty}\left(\mathbb{R}_{+},\{1, \ldots, N\}\right)$ but we assume that there exists a sequence $\left(\left[a_{n}, a_{n+1}\right)\right)_{n \geqslant 0}$ of consecutive intervals $\left(0=a_{0}<a_{1}<a_{2}<\cdots\right)$ whose union is equal to $\mathbb{R}_{+}$and such that for every index $n$, there exists an index $i_{n} \in\{1, \ldots, N\}$ with $\alpha_{i_{n}}(t)=1$ if $t \in\left[a_{n}, a_{n+1}\right)$ (informally, the $a_{i}$ 's are the switching instants). In what follows, we use the symbol $\delta_{n}$ to denote the length of $\left[a_{n-1}, a_{n}\right)$ with the convention that $\delta_{0}=0$.

Moreover, we shall deal with the scalar product related to matrix $P$ : if $x, y \in \mathbb{R}^{d}$, we put $\langle x, y\rangle=x^{\mathrm{T}} P y$; also we shall denote by $\|\cdot\|$ the related norm. We shall use the following result see $[25]$.

Theorem 1.1. If $A_{i}$ satisfies (1.2), then we can split $\mathbb{R}^{d}$ as $\mathbb{R}^{d}=V_{1}^{i} \oplus V_{2}^{i}$ where $V_{1}^{i}$ and $V_{2}^{i}$ are orthogonal and $A_{i}$-invariant, $A_{i}$ when restricted to $V_{1}^{i}$ has all its eigenvalues with negative real parts and $A_{i}$ restricted to $V_{2}^{i}$ is skew-symmetric (with respect to the scalar product $\langle\cdot, \cdot\rangle$ ).

In what follows, we assume that the skew-symmetric parts of matrices $A_{i}$ are zero. In other words, if $\mathcal{B}_{k}^{i}$ denotes a basis of $V_{k}^{i}(k=1,2)$, in basis $\mathcal{B}_{1}^{i} \cup \mathcal{B}_{2}^{i}$, matrix $A_{i}$ has the following representation

$$
A_{i}=\left(\begin{array}{cc}
A_{11}^{i} & 0 \\
0 & 0
\end{array}\right)
$$

where $A_{11}^{i}$ is a matrix of full rank. We denote by 1.2 the following assumption.

Assumption 1.2. There exists a positive definite matrix $P$ such that all matrices in family $\mathscr{F}$ satisfy relations (1.2) and (1.4).

Hereafter, we give the precise definition of the $\omega$-limit sets related to system (1.3).

Definition 1.3. We shall say that $\ell$ is an $\omega$-limit point of system (1.3) if there exists a sequence $\left(t_{n}\right)_{n \in \mathbb{N}}$ tending to infinity such that $\ell=\lim _{n \rightarrow+\infty} x\left(t_{n}\right)$. We denote by $\Omega\left(x_{0}\right)$ the set of $\omega$-limit points of system (1.3) issued from $x_{0}$.

\subsection{Preliminaries: paracontracting linear maps}

We denote by $p_{i}$ (resp. $q_{i}$ ) the orthogonal projection on $V_{1}^{i}\left(\right.$ resp. on $V_{2}^{i}$ ); obviously $p_{i}+q_{i}=\mathrm{Id}$. According Theorem 1.1, we can write

$$
e^{t A_{i}}(x)=e^{t A_{i}} \circ p_{1}(x)+q_{1}(x)
$$

and

$$
\left\|e^{t A_{i}}(x)\right\|^{2}=\left\|e^{t A_{i}} \circ p_{1}(x)\right\|^{2}+\left\|q_{1}(x)\right\|^{2} .
$$

Following [21] we introduce the notion of paracontracting maps. 
Definition 1.4. A linear mapping $f: \mathbb{R}^{d} \rightarrow \mathbb{R}^{d}$ is said to be paracontracting with respect to the norm $\|\cdot\|$ if $\|f(x)\| \leqslant\|x\|$ for every $x \in \mathbb{R}^{d}$ and $\|f(x)\|=\|x\|$ iff $f(x)=x$.

A family of matrices as described in the introduction generates a paracontracting semigroup. More precisely, we have the following result.

Lemma 1.5. If matrix $A$ is of the same type as (1.4), $e^{t A}$ is paracontracting for every $t \geqslant 0$.

Proof. If $t=0$, the result is obvious; in what follows, we shall assume that $t>0$. According to formula (1.6), one infers that

$$
\left\|e^{t A}(x)\right\|^{2} \leqslant\left\|p_{1}(x)\right\|^{2}+\left\|q_{1}(x)\right\|^{2}=\|x\|^{2} .
$$

Moreover, $\left\|e^{t A} x\right\|=\|x\|$ implies that $\left\|e^{t A} \circ p_{1}(x)\right\|^{2}=\left\|p_{1}(x)\right\|^{2}$. As $\left\|e^{s A} x\right\| \leqslant\left\|e^{s^{A} A} x\right\|$ if $s \leqslant s^{\prime}$, we have $\left\|e^{s A} \circ p_{1}(x)\right\|=\left\|p_{1}(x)\right\|$ for every $s \in[0, t]$. As the mapping $s \mapsto\left\|e^{s A} \circ p_{1}(x)\right\|^{2}$ is analytic, this implies that $\left\|e^{s A} \circ p_{1}(x)\right\|=\left\|p_{1}(x)\right\|$ for every $s \geqslant 0$, hence $p_{1}(x)$ is in ker $A$ and so $p_{1}(x)$ must be zero and $e^{t A} x=x$ follows from formula (1.5).

This lemma allows us to state the following easy result.

Proposition 1.6. The elements of $\Omega\left(x_{0}\right)$ are of the same norm, in other words, there exists $r \geqslant 0$ such that, $\Omega\left(x_{0}\right)$ is included in the sphere centered at the origin of $\mathbb{R}^{d}$ and with radius $r$.

Proof. Let $\ell$ and $\ell^{\prime}$ be two elements in $\Omega\left(x_{0}\right)$. There exist two sequences $\left(t_{n}\right)_{n \in \mathbb{N}}$ and $\left(t_{n}^{\prime}\right)_{n \in \mathbb{N}}$ such that $\ell=\lim _{n \rightarrow+\infty} x\left(t_{n}\right)$ and $\ell^{\prime}=\lim _{n \rightarrow+\infty} x\left(t_{n}^{\prime}\right)$. For each $n$, there exists $m_{n}$ such that $t_{n} \leqslant t_{m_{n}}^{\prime}$, so $x\left(t_{m_{n}}^{\prime}\right)=\varphi_{n}\left(x\left(t_{n}\right)\right)$ where $\varphi_{n}$ is a product of exponentials of matrices taken in $\left\{A_{1}, \ldots, A_{N}\right\}$. As these exponentials are paracontracting, we have $\left\|x\left(t_{m_{n}}^{\prime}\right)\right\| \leqslant\left\|x\left(t_{n}\right)\right\|$ and passing to the limit as $n \rightarrow+\infty$, we get $\left\|\ell^{\prime}\right\| \leqslant\|\ell\|$ and we can prove the opposite inequality in the same way.

Finally, the following elementary result, given without proof, will be useful for the proofs of results given in the next section.

Proposition 1.7. If matrix $A$ is of the same type as in (1.4), and if $x \notin \operatorname{ker} A$, then for all $\tau>0$, there exists $\rho \in(0,1)$ such that $t \geqslant \tau$ implies $\left\|e^{t A} x\right\| \leqslant \rho\|x\|$.

\section{A condition for the convergence to zero}

In this section, we deal with the problem of the convergence to zero of the solution of system (1.3). First an easy remark: if the intersection $\bigcap_{i=1}^{N}$ ker $A_{i}$ is not $\{0\}$, then, taking as an initial condition for (1.3) a nonzero element $x_{0}$ of this intersection, the solution $x(t)$ is constant and equal to $x_{0}$. So, we introduce this first assumption

Assumption 2.1. We say that system (1.3) satisfies the null intersection assumption if $\bigcap_{i=1}^{N} \operatorname{ker} A_{i}=\{0\}$.

As we shall see through the following example, Assumption 2.1 is not sufficient to ensure the convergence to zero.

Example 2.2. In $\mathbb{R}^{3}$, we consider the following matrices

$$
A_{1}=\left(\begin{array}{ccc}
0 & -1 & a_{13} \\
1 & 0 & a_{23} \\
-a_{13} & -a_{23} & a_{33}
\end{array}\right) \quad A_{2}=\left(\begin{array}{ccc}
0 & -1 & -a_{13} \\
1 & 0 & -a_{23} \\
a_{13} & a_{23} & a_{33}
\end{array}\right)
$$


with $a_{33}<0$ and one of the coefficients $a_{13}$ or $a_{23}$ different from zero. Denoting by $\langle\cdot, \cdot\rangle$ the canonical scalar product in $\mathbb{R}^{3}$, we have $\left\langle A_{i} x, x\right\rangle=a_{33} x_{3}^{2} \leqslant 0(i=1,2)$ for every $x \in \mathbb{R}^{3}$. Moreover the characteristic polynomials of $A_{1}$ and $A_{2}$ are both equal to

$$
-X^{3}+a_{33} X^{2}-\left(a_{13}^{2}+a_{23}^{2}+1\right) X+a_{33}
$$

so, we can see that $A_{1}$ and $A_{2}$ do not have purely imaginary eigenvalues. We can conclude that these matrices satisfy assumption 1.2. We shall use now the well known Trotter's formula (see [15] or [14], p. 78 and ff.):

$$
\lim _{n \rightarrow+\infty}\left(e^{t_{0} M / n} \circ e^{t_{0} N / n}\right)^{n}=e^{t_{0}(M+N)}
$$

where $M$ and $N$ are squared matrices and $t_{0}$ is a real number. Define the sequence $\left(e_{k}\right)_{k \in \mathbb{N}} \subset \mathbb{R}^{3}$ by: $e_{0}=(1,0,0)^{\mathrm{T}}, e_{1}=(0,1,0)^{\mathrm{T}}, e_{2}=(-1,0,0)^{\mathrm{T}}, e_{3}=(0,-1,0)^{\mathrm{T}}$ and for $k \geqslant 4, e_{k}$ will refer to vector $e_{r}$ where $r$ is the remainder in the Euclidean division of $k$ by 4 . We have

$$
A_{1}+A_{2}=\left(\begin{array}{ccc}
0 & -2 & 0 \\
2 & 0 & 0 \\
0 & 0 & 2 a_{33}
\end{array}\right)
$$

and so, if we take $t_{0}=\pi / 4$, we have $e^{t_{0}\left(A_{1}+A_{2}\right)}\left(e_{k}\right)=e_{k+1}$. Set

$$
R=e^{t_{0}\left(A_{1}+A_{2}\right)} \quad \text { and } \quad \varphi_{n}=\left(e^{t_{0} A_{1} / n} \circ e^{t_{0} A_{2} / n}\right)^{n} .
$$

Take $\varepsilon>0$ and $x_{0}=e_{0}$, from formula (2.1), we know that there exists $n_{1}$ such that

$$
\left\|\varphi_{n_{1}}\left(x_{0}\right)-R\left(x_{0}\right)\right\| \leqslant \frac{\varepsilon}{2} .
$$

Assume now that we have built a sequence $\left(x_{1}, \ldots, x_{k}\right)$ and we have found integers $\left(n_{1}, \ldots, n_{k}\right)$ such that $x_{p}=\varphi_{n_{p}}\left(x_{p-1}\right)$ and $\left\|x_{p}-R\left(x_{p-1}\right)\right\| \leqslant \varepsilon / 2^{p}$ for $p=1, \ldots, k$. Take $n_{k+1}$ such that $\left\|\varphi_{n_{k+1}}\left(x_{k}\right)-R\left(x_{k}\right)\right\| \leqslant \varepsilon / 2^{k+1}$ and set $x_{k+1}=\varphi_{n_{k+1}}\left(x_{k}\right)$. Consider the sequences $\left(x_{k}\right)_{k \geqslant 1}$ and $\left(n_{k}\right)_{k \geqslant 1}$. Inequality $(2.2)$ can be written $\left\|x_{1}-e_{1}\right\| \leqslant \varepsilon / 2$ and we shall prove by induction that

$$
\left\|x_{k}-e_{k}\right\| \leqslant \frac{\varepsilon}{2^{k}}+\cdots+\frac{\varepsilon}{2} \text {. }
$$

We have

$$
\begin{aligned}
\left\|x_{k+1}-e_{k+1}\right\| & \leqslant\left\|x_{k+1}-R\left(x_{k}\right)\right\|+\left\|R\left(x_{k}\right)-R\left(e_{k}\right)\right\| \\
& \leqslant\left\|\varphi_{n_{k+1}}\left(x_{k}\right)-R\left(x_{k}\right)\right\|+\left\|x_{k}-e_{k}\right\| \\
& \leqslant \frac{\varepsilon}{2^{k+1}}+\frac{\varepsilon}{2^{k}}+\cdots \frac{\varepsilon}{2}
\end{aligned}
$$$$
\text { because }\|R\| \leqslant 1
$$$$
\text { by induction hypothesis. }
$$

From (2.3), we get $\left\|x_{k}-e_{k}\right\| \leqslant \varepsilon$ for every integer $k$. Consider the switch law $\alpha=\left(\alpha_{1}, \alpha_{2}\right)$ defined by

$$
\begin{aligned}
& \alpha_{1}(t)= \begin{cases}1 & \text { if } t \in\left[(k-1) t_{0}+\left(2 p / n_{k}\right) t_{0},(k-1) t_{0}+\left((2 p+1) / n_{k}\right) t_{0}\right) \\
0 & \text { otherwise }\end{cases} \\
& \alpha_{2}(t)=1-\alpha_{1}(t) .
\end{aligned}
$$

where $k$ and $p$ are integers such that $2 p<n_{k}$. The switched system defined by matrices $A_{1}$ and $A_{2}$ and laws $\alpha_{1}$ and $\alpha_{2}$ is such that $\Omega\left(e_{0}\right)$ is included in the exterior of the circle of center 0 with radius $1-\varepsilon$ and so $0 \notin \Omega\left(e_{0}\right)$ if $\varepsilon$ is chosen small enough. Moreover $\Omega\left(e_{0}\right)$ contains at least one point in every open ball $B\left(e_{i}, \varepsilon\right)$ and so, being connected, $\Omega\left(e_{0}\right)$ is an infinite set. 
We state the following definition.

Definition 2.3. We shall say that the finite sequence $\left(i_{1}, \ldots, i_{p}\right)$ of indices taken in $\{1, \ldots, N\}$ is compatible with system (1.3) if there exists $p$ consecutive intervals $\left[a_{n}, a_{n+1}\right), \ldots,\left[a_{n+p-1}, a_{n+p}\right)$ such that for every $q \in\{1, \ldots, p\}, \alpha_{i_{q}}(t)=1$ if $t \in\left[a_{n+q-1}, a_{n+q}\right)$.

We introduce now the following assumptions:

Assumption 2.4 (Dwell-time). We shall say that the mode $i$ satisfies the condition of dwell time if there exists a positive number $\tau$ and an integer $p$ such that for every compatible sequence $\left(i_{1}, \ldots, i_{p}\right)$ there exists an index $q \in\{1, \ldots, p\}$ with $i_{q}=i$ and, if $\left[a_{n}, a_{n+1}\right), \ldots,\left[a_{n+p-1}, a_{n+p}\right)$ is the related sequence of consecutive intervals, $a_{n+q}-a_{n+q-1} \geqslant \tau$.

We state the following result concerning the modes satisfying the dwell-time condition.

Theorem 2.5. If assumptions 2.4 is satisfied for the mode $i$, then every point of the $\omega$-limit set $\Omega\left(x_{0}\right)$ belongs to the kernel of matrix $A_{i}$.

Proof. Without loss of generality, we may assume that the dwell time assumption is satisfied for mode $i=1$. The solution of system (1.3) at time $t \in\left[a_{n}, a_{n+1}\right)$ reads

$$
x(t)=e^{\left(t-a_{n}\right) A_{i_{n+1}}} \circ e^{\delta_{n} A_{i_{n}}} \circ \cdots \circ e^{\delta_{1} A_{i_{1}}}\left(x_{0}\right) .
$$

Let $\ell \in \Omega\left(x_{0}\right)$, there exists a sequence $\left(t_{k}\right)_{k \in \mathbb{N}}$ such that $\ell=\lim _{k \rightarrow+\infty} x\left(t_{k}\right)$. For every $k, t_{k}$ belongs to an interval $\left[a_{n_{k}}, a_{n_{k}+1}\right)$ and there exists an interval $\left[a_{m_{k}}, a_{m_{k}+1}\right)$ with $a_{m_{k}}>a_{n_{k}}$ and such that $\alpha_{1}(t)=1$ if $t \in\left[a_{m_{k}}, a_{m_{k}+1}\right)$ and $\delta_{m_{k}+1} \geqslant \tau$. Due to Assumption 2.4, the sequence of integers $\left(m_{k}-n_{k}\right)_{k \geqslant 1}$ is bounded and so even if we have to work with a subsequence of $\left(t_{k}\right)_{k \in \mathbb{N}}$, we can assume that

- the difference $m_{k}-n_{k}$ is constant (positive), we denote by $r$ this difference,

- the sequence of switches from $t_{k}$ to $a_{m_{k}}$ is independent of $k$, that is to say, there exists a finite sequence $A_{i_{1}}, \ldots, A_{i_{r}}$ of matrices taken in $\left\{A_{1}, \ldots, A_{N}\right\}$ such that for every $k$

$$
x\left(a_{m_{k}}\right)=e^{u_{k}^{r} A_{i_{r}}} \circ \cdots \circ e^{u_{k}^{1} A_{i_{1}}}\left(x\left(t_{k}\right)\right),
$$

- for each $m=1, \ldots, r$, or the sequence $\left(u_{k}^{m}\right)_{k \in \mathbb{N}}$ tends to zero as $k$ tends to infinity either there exists $v^{m}>0$ such that $u_{k}^{m} \geqslant v^{m}$ for all $k$.

We first prove by induction on $r$ that $\lim _{k \rightarrow+\infty} x\left(a_{m_{k}}\right)=\ell$. If $r=1$, we have $x\left(a_{m_{k}}\right)=$ $e^{u_{k}^{1} A_{i_{1}}}\left(x\left(t_{k}\right)\right)$. If $\lim _{k \rightarrow+\infty} u_{k}^{1}=0$, the result is obvious, if not, write

$$
x\left(t_{k}\right)=p_{i_{1}}(\ell)+q_{i_{1}}(\ell)+\bar{x}_{k},
$$

so we have

$$
e^{u_{k}^{1} A_{i_{1}}}\left(x\left(t_{k}\right)\right)=e^{u_{k}^{1} A_{i_{1}}}\left(p_{i_{1}}(\ell)\right)+q_{i_{1}}(\ell)+e^{u_{k}^{1} A_{i_{1}}}\left(\bar{x}_{k}\right),
$$

and, as $V_{1}^{i_{1}}$ and $V_{2}^{i_{1}}$ are orthogonal

$$
\left\|e^{u_{k}^{1} A_{i_{1}}}\left(x\left(t_{k}\right)\right)\right\|^{2}=\left\|e^{u_{k}^{1} A_{i_{1}}}\left(p_{i_{1}}(\ell)\right)\right\|^{2}+\left\|q_{i_{1}}(\ell)\right\|^{2}+2\left\langle e^{u_{k}^{1} A_{i_{1}}}(\ell), e^{u_{k}^{1} A_{i_{1}}}\left(\bar{x}_{k}\right)\right\rangle+\left\|e^{u_{k}^{1} A_{i_{1}}}\left(\bar{x}_{k}\right)\right\|^{2} .
$$

Suppose, to reach a contradiction, that $p_{i_{1}}(\ell) \neq 0$. Then, as $u_{k}^{1} \geqslant v^{1}$, there exists $\rho \in(0,1)$ such that $\left\|e^{u_{k}^{1} A_{i_{1}}}\left(p_{i_{1}}(\ell)\right)\right\|<\rho\left\|p_{i_{1}}(\ell)\right\|$. Taking into account that $\left\|e^{u_{k}^{1} A_{i_{1}}}\right\| \leqslant 1$, we get

$$
\left\|e^{u_{k}^{1} A_{i_{1}}}\left(x\left(t_{k}\right)\right)\right\|^{2}<\rho^{2}\left\|p_{i_{1}}(\ell)\right\|^{2}+\left\|q_{i_{1}}(\ell)\right\|^{2}+2\|\ell\|\left\|\bar{x}_{k}\right\|+\left\|\bar{x}_{k}\right\|^{2} .
$$


Choose $\varepsilon>0$, as $\lim _{k \rightarrow+\infty} \bar{x}_{k}=0$, we have

$$
\left\|e^{u_{k}^{1} A_{i_{1}}}\left(x\left(t_{k}\right)\right)\right\|^{2}<\rho^{2}\left\|p_{i_{1}}(\ell)\right\|^{2}+\left\|q_{i_{1}}(\ell)\right\|^{2}+2 \varepsilon\|\ell\|+\varepsilon^{2}
$$

if $k$ is large enough. The right hand side of (2.4) can be made less than $\|\ell\|^{2}-\varepsilon^{2}$ provided that $\varepsilon$ is chosen such that $2 \varepsilon^{2}+2 \varepsilon\|\ell\|<\left(1-\rho^{2}\right)\left\|p_{i_{1}}(\ell)\right\|^{2}$. But in this case, we could find an element in $\Omega\left(x_{0}\right)$ (a cluster point of the sequence $\left(x\left(a_{m_{k}}\right)\right)_{k \geqslant 1}$ ) whose norm is less than $\|\ell\|$, which is impossible. Hence, $p_{i_{1}}(\ell)=0$ which implies $\ell=q_{i_{1}}(\ell)$ and $e^{u_{k}^{1} A_{i_{1}}}\left(x\left(t_{k}\right)\right)=\ell+e^{u_{k}^{1} A_{i_{1}}}\left(\bar{x}_{k}\right)$, since $\left\|e^{u_{k}^{1} A_{i_{1}}}\right\| \leqslant 1$ and $\lim _{k \rightarrow+\infty} \bar{x}_{k}=0$, we get the result. So, we can write

$$
x\left(a_{m_{k}}\right)=e^{u_{k}^{r} A_{i_{r}}} \circ \cdots \circ e^{u_{k}^{2} A_{i_{2}}}\left(x\left(a_{n_{k}}\right)\right)
$$

with $\lim _{k \rightarrow+\infty} x\left(a_{n_{k}}\right)=\ell$, applying the induction hypothesis, we conclude that $\lim _{k \rightarrow+\infty} x\left(a_{m_{k}}\right)=$ $\ell$. Now, we have

$$
x\left(a_{m_{k}+1}\right)=e^{\left(a_{m_{k}+1}-a_{m_{k}}\right) A_{1}}\left(x\left(a_{m_{k}}\right)\right)
$$

with $a_{m_{k}+1}-a_{m_{k}} \geqslant \tau$. The argument used above in the case where the sequence $\left(u_{k}^{1}\right)_{k \in \mathbb{N}}$ does not converge to zero proves that $p_{1}(\ell)=0$, i.e., $\ell \in \operatorname{ker} A_{1}$.

An easy consequence of Theorem 2.5 is stated in the following corollary.

Corollary 2.6. If system (1.3) satisfies Assumption 2.1 and if every mode satisfies assumption 2.4, then $x(t)$, the solution of system (1.3), tends to zero as $t$ tends to infinity.

Proof. According to Theorem 2.5 and Assumption 2.1, we have $\Omega\left(x_{0}\right) \in \bigcap_{i=1}^{N} \operatorname{ker} A_{i}=\{0\}$.

At this step, we could wonder if it were possible to weaken the hypothesis of Theorem 2.5. Consider the following hypothesis which is weaker than assumption 2.4.

Assumption 2.7 (Weak dwell-time). We shall say that the mode $i$ satisfies the weak dwelltime condition if there exists $\tau>0$ such that for every $n_{0} \in \mathbb{N}$, there exists $n \geqslant n_{0}$ with $\alpha_{i}(t)=1$ if $t \in\left[a_{n}, a_{n+1}\right)$ and $a_{n+1}-a_{n} \geqslant \tau$.

To say that assumption 2.7 is satisfied amounts to say that the sequence of durations during which mode $i$ is activated does not tends to zero. As we shall see through the following example, Assumption 2.4 cannot be replaced by Assumption 2.7 in Theorem 2.5.

Example 2.8. In the following family of eight matrices, we assume that the diagonal coefficients $a_{11}, \ldots, a_{44}$ are negative, the nondiagonal coefficients $a_{i j}(i \neq j)$ being nonzero.

$$
\begin{aligned}
A_{1} & =\left(\begin{array}{cccc}
0 & -1 & a_{13} & 0 \\
1 & 0 & a_{23} & 0 \\
-a_{13} & -a_{23} & a_{33} & 0 \\
0 & 0 & 0 & 0
\end{array}\right), & A_{2} & =\left(\begin{array}{cccc}
0 & -1 & -a_{13} & 0 \\
1 & 0 & -a_{23} & 0 \\
a_{13} & a_{23} & a_{33} & 0 \\
0 & 0 & 0 & 0
\end{array}\right), \\
A_{3} & =\left(\begin{array}{cccc}
0 & 0 & 0 & 0 \\
0 & 0 & -1 & a_{24} \\
0 & 1 & 0 & a_{34} \\
0 & -a_{24} & -a_{34} & a_{44}
\end{array}\right), & A_{4} & =\left(\begin{array}{cccc}
0 & 0 & 0 & 0 \\
0 & 0 & -1 & -a_{24} \\
0 & 1 & 0 & -a_{34} \\
0 & a_{24} & a_{34} & a_{44}
\end{array}\right), \\
A_{5} & =\left(\begin{array}{cccc}
a_{11} & 0 & a_{13} & a_{14} \\
0 & 0 & 0 & 0 \\
-a_{13} & 0 & 0 & -1 \\
-a_{14} & 0 & 1 & 0
\end{array}\right), & A_{6} & =\left(\begin{array}{cccc}
a_{11} & 0 & -a_{13} & -a_{14} \\
0 & 0 & 0 & 0 \\
a_{13} & 0 & 0 & -1 \\
a_{14} & 0 & 1 & 0
\end{array}\right),
\end{aligned}
$$




$$
A_{7}=\left(\begin{array}{cccc}
0 & a_{12} & 0 & -1 \\
-a_{12} & a_{22} & 0 & a_{24} \\
0 & 0 & 0 & 0 \\
1 & -a_{24} & 0 & 0
\end{array}\right), \quad A_{8}=\left(\begin{array}{cccc}
0 & -a_{12} & 0 & -1 \\
a_{12} & a_{22} & 0 & -a_{24} \\
0 & 0 & 0 & 0 \\
1 & a_{24} & 0 & 0
\end{array}\right)
$$

As in Example 2.2, we can easily check that these matrices satisfy Assumption 1.2. In this example, $e_{1}, \ldots, e_{4}$ will denote the vectors of the canonical basis in $\mathbb{R}^{4}$ and $R_{1}, \ldots, R_{4}$ will denote the matrices $R_{i}=e^{t_{0}\left(A_{2 i-1}+A_{2 i}\right)}(i=1, \ldots, 4)$. Taking $t_{0}=\pi / 4$, we clearly have $R_{i}\left(e_{i}\right)=e_{i+1}$ for $i=1, \ldots, 4$ (where $e_{5} \triangleq e_{4}$ ). Finally, we denote by $\varphi_{n}^{i}(i=1, \ldots, 4)$ the mappings $\varphi_{n}^{i}=\left(e^{\left(t_{0} / n\right) A_{2 i-1}} \circ e^{\left(t_{0} / n\right) A_{2 i}}\right)^{n}$ and we notice that, following the Trotter's formula, we have $\lim _{n \rightarrow+\infty} \varphi_{n}^{i}=R_{i}$. Choose $\tau>0$ and consider the following product of matrices

$$
P_{1}=\prod_{i=5}^{8} e^{\tau A_{2 i+4}} \circ \varphi_{n_{i}}^{i-4} \circ \prod_{i=1}^{4} e^{\tau A_{2 i+3}} \circ \varphi_{n_{i}}^{i}
$$

where we make the convention that if $j$ is greater than 8, matrix $A_{j}$ is equal to $A_{r}$ where $r$ is the remainder in the Euclidean division of $j$ by 8 excepted when $r=0$ in which case, $A_{j}$ is $A_{8}$. We proceed as in Example 2.2. Choose $\varepsilon>0$ and $n_{1}$ such that $\left\|\varphi_{n_{1}}^{1}\left(e_{1}\right)-R_{1}\left(e_{1}\right)\right\| \leqslant \varepsilon / 4$. Then, we have

$$
\left\|e^{\tau A_{5}} \circ \varphi_{n_{1}}^{1}\left(e_{1}\right)-e^{\tau A_{5}} \circ R_{1}\left(e_{1}\right)\right\| \leqslant \varepsilon / 4
$$

because $\left\|e^{\tau A_{5}}\right\| \leqslant 1$. Notice that, in this inequality, $e^{\tau A_{5}} \circ R_{1}\left(e_{1}\right)=e_{2}$. Then, choose $n_{2}$ such that

$$
\left\|\varphi_{n_{2}}^{2} \circ e^{\tau A_{5}} \circ \varphi_{n_{1}}^{1}\left(e_{1}\right)-R_{2} \circ e^{\tau A_{5}} \circ \varphi_{n_{1}}^{1}\left(e_{1}\right)\right\| \leqslant \varepsilon / 8
$$

and we have

$$
\begin{aligned}
\left\|\varphi_{n_{2}}^{2} \circ e^{\tau A_{5}} \circ \varphi_{n_{1}}^{1}\left(e_{1}\right)-e_{3}\right\| \leqslant & \left\|\varphi_{n_{2}}^{2} \circ e^{\tau A_{5}} \circ \varphi_{n_{1}}^{1}\left(e_{1}\right)-R_{2} \circ e^{\tau A_{5}} \circ \varphi_{n_{1}}^{1}\left(e_{1}\right)\right\| \\
& +\left\|R_{2} \circ e^{\tau A_{5}} \circ \varphi_{n_{1}}^{1}\left(e_{1}\right)-R_{2} \circ e^{\tau A_{5}} \circ R_{1}\left(e_{1}\right)\right\| \\
\leqslant & \varepsilon / 8+\left\|e^{\tau A_{5}} \circ \varphi_{n_{1}}^{1}\left(e_{1}\right)-e^{\tau A_{5}} \circ R_{1}\left(e_{1}\right)\right\| \\
\leqslant & \varepsilon / 8+\varepsilon / 4 .
\end{aligned}
$$

Proceeding this way, we choose $n_{3}, \ldots, n_{8}$ such that $\left\|P_{1}\left(e_{1}\right)-e_{1}\right\| \leqslant \frac{\varepsilon}{2^{9}}+\cdots+\frac{\varepsilon}{4}<\frac{\varepsilon}{2}$. We then build $P_{2}, P_{3}, \ldots$ in the same way as $P_{1}$ and we choose the integers $n_{9}, n_{10}, \ldots$ in such a way that $\left\|P_{k}\left(e_{1}\right)-e_{1}\right\| \leqslant \varepsilon / 2^{k}$. Now reasoning as in Example 2.2, we can build a switch law related to the family of matrices $A_{1}, \ldots, A_{8}$ such that $x\left(8 k\left(t_{0}+\tau\right)\right)=P_{k} \circ \cdots \circ P_{1}\left(e_{1}\right)$. The $\omega$-limit set $\Omega\left(e_{1}\right)$ contains at last one point in the open ball $B\left(e_{1}, \varepsilon\right)$; this ball does not contain the origin of $\mathbb{R}^{4}$ if $\varepsilon$ is chosen small enough and so $x(t)$ does not tend to zero as $t$ tends to infinity. Nevertheless, every mode of this system satisfies assumption 2.7 .

This example shows that in Assumption 2.4, one cannot cancel the condition on the repartition of switches (excepted in the two-dimensional case as we shall see in the next section). Nevertheless, if we make a stronger assumption on the dwell-times, we can free ourselves from this condition; consider the following assumption.

Assumption 2.9 (Strong dwell-time). We shall say that the mode $i$ satisfies the condition of strong dwell time if there exists $\tau>0$ such that for every $n_{i} \in \mathbb{N}$ satisfying $\left.\alpha_{i}\right|_{\left[a_{n_{i}}, a_{n_{i}+1}\right)}=1$, we have $a_{n_{i}+1}-a_{n_{i}} \geqslant \tau$. In other words, the sequence of durations during which the mode $i$ is activated has a positive inferior limit.

We have the following result. 
Theorem 2.10. If system (1.3) satisfies Assumptions 2.1 and 2.9, then $\Omega\left(x_{0}\right)=\{0\}$ for every $x_{0} \in \mathbb{R}^{d}$.

Proof. We let $t_{n}=\sum_{k=1}^{n} \delta_{k}$. As the solution $x(t)$ of (1.3) is bounded, to get the result, it is sufficient to prove that $\Omega\left(x_{0}\right)=\{0\}$. To this end, we have only to prove that every cluster point of the sequence $\left(x\left(t_{n}\right)\right)_{n \in \mathbb{N}}$ is equal to zero. Let $\ell$ be such a point, then, $\ell$ is the limit of a subsequence $\left(x\left(t_{n_{k}}\right)\right)_{k \in \mathbb{N}}$ of $\left(x\left(t_{n}\right)\right)_{n \in \mathbb{N}}$. Write $x\left(t_{n_{k}}\right)=e^{\delta_{n_{k}} A\left(n_{k}\right)}\left(x\left(t_{n_{k}-1}\right)\right)$, where $A\left(n_{k}\right)$ is a matrix in family $\mathscr{F}$. There exists an index $i \in\{1, \ldots, N\}$ such that, for infinitely many indices $n_{k}$, we have $A\left(n_{k}\right)=A_{i}$. Even if we have to renumber the matrices of family $\mathscr{F}$, we can suppose that $i=1$ and, even if we have to work with a subsequence of $\left(x\left(t_{n_{k}}\right)\right)_{k \in \mathbb{N}}$, we can write $x\left(t_{n_{k}}\right)=e^{\delta_{n_{k}} A_{1}}\left(x\left(t_{n_{k}-1}\right)\right)$. We write $x\left(t_{n_{k}-1}\right)=\ell+\bar{x}_{k}$ and, in exactly the same manner as in the proof of Theorem 2.5, we prove that $p_{1}(\ell)=0$.

We now make the following induction hypothesis: $p_{1}(\ell)=\cdots=p_{r-1}(\ell)=0$. For infinitely many indices $k$, we can find in the sequence $\left(x\left(t_{n_{k}}\right)\right)_{k \in \mathbb{N}}$, terms which write $x\left(t_{m_{k}}\right)=e^{\delta_{m_{k}} A_{r}}$ o $\varphi_{k}\left(x\left(t_{n_{k}-1}\right)\right)$ where $\varphi_{k}$ is a product of exponentials of matrices taken in the set $\left\{A_{1}, \ldots, A_{r-1}\right\}$. Writing $x\left(t_{n_{k}-1}\right)=\ell+\bar{x}_{k}$, we have $\varphi_{k}\left(x\left(t_{n_{k}-1}\right)\right)=\ell+\varphi_{k}\left(\bar{x}_{k}\right)$ because $\ell \in \bigcap_{i=1}^{r-1} \operatorname{ker} A_{i}$. Thus, $x\left(t_{m_{k}}\right)=e^{\delta_{m_{k}} A_{r}}(\ell)+e^{\delta_{m_{k}} A_{r}}\left(\varphi_{k}\left(\bar{x}_{k}\right)\right)$. Since $\lim _{k \rightarrow+\infty} e^{\delta_{m_{k}} A_{r}}\left(\varphi_{k}\left(\bar{x}_{k}\right)\right)=0$, if we suppose that $p_{r}(\ell) \neq 0$, we are led to a contradiction in the same way as above. We have thus proved that $p_{1}(\ell)=\cdots=p_{N}(\ell)=0$, or equivalently, that $\ell \in \bigcap_{i=1}^{N}$ ker $A_{i}$. According to Assumption 2.1, this set is $\{0\}$ and so $\ell=0$.

\section{Some properties of the $\omega$-limit set}

\subsection{General considerations}

In this section, we introduce the following new assumption.

Assumption 3.1 (Permanent excitation). We say that the mode $i$ satisfies the permanent excitation hypothesis if $\lambda\left(\left\{t \geqslant 0 \mid \alpha_{i}(t)=1\right\}\right)=+\infty$.

We begin by proving an easy result.

Proposition 3.2. A control law which satisfies Assumption 3.1 for every mode being given, the set of points $x_{0}$ such that $\Omega\left(x_{0}\right)$ is equal to $\{0\}$ is a subspace of $\mathbb{R}^{d}$ with dimension at least one. This implies that the set of points $x_{0}$ such that $\Omega\left(x_{0}\right)$ does not reduce to $\{0\}$ is either empty or open and dense.

Proof. Obviously the set of points $x_{0}$ such that $\Omega\left(x_{0}\right)=\{0\}$ is a subspace of $\mathbb{R}^{d}$; moreover, $x\left(t, x_{0}\right)$, the solution of (1.3) issued from $x_{0}$ can be written as $x\left(t, x_{0}\right)=\varphi(t)\left(x_{0}\right)$ where $\varphi(t)$ is a product of exponentials of matrices taken in the set $\left\{A_{1}, \ldots, A_{N}\right\}$. The determinant of $\varphi(t)$ is equal to $\operatorname{det} \varphi(t)=e^{\tau_{1}(t) \operatorname{tr} A_{1}} \cdots e^{\tau_{N}(t) \operatorname{tr} A_{N}}$, where $\tau_{i}(t)$ denotes the measure of the set $\left\{0 \leqslant s \leqslant t \mid \alpha_{i}(s)=1\right\}\left(\operatorname{tr} A_{i}<0\right)$. Due to Assumption 3.1, we have $\lim _{t \rightarrow+\infty} \tau_{i}(t)=+\infty$, and so $\lim _{t \rightarrow+\infty} \operatorname{det} \varphi(t)=0$. As, $\varphi(t)$ is bounded, we can find a sequence $\left(t_{n}\right)_{n \in \mathbb{N}}$ such that the limit $\lim _{n \rightarrow+\infty} \varphi\left(t_{n}\right)$ exists. Let us denote by $\phi$ this limit, as $\operatorname{det} \phi=0$, there exists $x_{0} \neq 0$ such that $\phi\left(x_{0}\right)=0$; for this $x_{0}$, we clearly have $\Omega\left(x_{0}\right)=\{0\}$.

We consider now the set $M$ related to family $\mathscr{F}$ defined as

$$
M=\left\{x \in \mathbb{R}^{n} \mid\left\langle A_{i} x, x\right\rangle=0, i=1, \ldots, N\right\} .
$$

For $i=1, \ldots, N$, we also denote by $M_{i}$ the set $M_{i}=\left\{x \in \mathbb{R}^{n} \mid\left\langle A_{i} x, x\right\rangle=0\right\}$. Set $M$ can be regarded as the intersection of the sets of zeros of quadratic forms $x \mapsto\left\langle A_{i} x, x\right\rangle$ which are 
subspaces of $\mathbb{R}^{d}$ because these quadratic forms are non positive, so $M$ is a subspace of $\mathbb{R}^{d}$. Notice than $M$ can contain a nonzero vector even if all the matrices in family $\mathscr{F}$ are of full rank. For instance, consider the two matrices $A_{1}$ and $A_{2}$ of Example 2.2, the related set $M$ is $M=\left\{x \in \mathbb{R}^{3} \mid x_{3}=0\right\}$.

The two following propositions state that the set of $\omega$-limit points cannot avoid sets $M_{i}$.

Proposition 3.3. Suppose that system (1.3) satisfies Assumption 3.1 for mode $i$, then there exists $\ell \in \Omega\left(x_{0}\right)$ such that $\left\langle A_{i} \ell, \ell\right\rangle=0$ (in other words, $\ell \in M_{i}$ ).

Proof. The proof is by contradiction. Assume that for every $\ell \in \Omega\left(x_{0}\right)$, the scalar product $\left\langle A_{i} \ell, \ell\right\rangle$ is nonzero, it is therefore negative. As $\Omega\left(x_{0}\right)$ is compact, this implies that there exists $\mu>0$, such that $\left\langle A_{i} \ell, \ell\right\rangle \leqslant-\mu$ for every $\ell \in \Omega\left(x_{0}\right)$. We denote by $\left[a_{k_{1}}, a_{k_{1}+1}\right),\left[a_{k_{2}}, a_{k_{2}+1}\right), \ldots$ the intervals of times during which the mode $i$ is activated ${ }^{1}$. For the sake of readability, we denote by $x_{n}$ the solution of (1.3) at time $a_{k_{n}+1}$. We have

$$
x_{n}=e^{\delta_{k_{n}} A_{j}} \circ \varphi_{n}\left(x_{n-1}\right)
$$

where $\varphi_{n}$ is a product of exponentials of matrices taken in family $\mathscr{F} \backslash\left\{A_{i}\right\}$. It follows from $\left\|\varphi_{n}\right\| \leqslant 1$ that

$$
\begin{aligned}
\left\|x_{n}\right\|^{2}-\left\|x_{0}\right\|^{2} & =\sum_{k=1}^{n}\left(\left\|x_{k}\right\|^{2}-\left\|\varphi_{k}\left(x_{k-1}\right)\right\|^{2}\right)+\sum_{k=1}^{n}\left(\left\|\varphi_{k}\left(x_{k-1}\right)\right\|^{2}-\left\|x_{k-1}\right\|^{2}\right) \\
& \leqslant \sum_{k=1}^{n}\left(\left\|x_{k}\right\|^{2}-\left\|\varphi_{k}\left(x_{k-1}\right)\right\|^{2}\right)
\end{aligned}
$$

First, we show the result when $\lim _{n \rightarrow+\infty} \delta_{k_{n}}=0$. In this case, we consider the series whose general term is

$$
\left\|x_{n}\right\|^{2}-\left\|\varphi_{n}\left(x_{n-1}\right)\right\|^{2}=\left\|e^{\delta_{k_{n}} A_{1}} \circ \varphi_{n}\left(x_{n-1}\right)\right\|^{2}-\left\|\varphi_{n}\left(x_{n-1}\right)\right\|^{2} .
$$

If $n$ is large enough, the scalar product $\left\langle A_{i} x_{n}, x_{n}\right\rangle$ is far away from zero, more precisely, there exists $n_{0}$ such that $\left\langle A_{i} x_{n}, x_{n}\right\rangle \leqslant-\mu / 2$ as soon as $n \geqslant n_{0}$. So, as $n$ tends to infinity, the general term of this series is equivalent to $2\left\langle A_{i} x_{n}, x_{n}\right\rangle \delta_{k_{n}}$ which is the general term of a divergent (to $-\infty)$ series since the series whose general term is $\delta_{k_{n}}$ is divergent and $\left\langle A_{i} x_{n}, x_{n}\right\rangle \leqslant-\mu / 2$. So the right-hand side of inequality (3.2) can be made less than $\|\ell\|^{2}-\left\|x_{0}\right\|^{2}$ if $n$ is chosen large enough which is a contradiction since $\left\|x_{n}\right\| \geqslant\|\ell\|$ for all $n$.

In the case where we do not have $\lim _{n \rightarrow+\infty} \delta_{k_{n}}=0$, there exists $\tau>0$ such that for all $n_{0}$, there exists $n \geqslant n_{0}$ with $\delta_{k_{n}} \geqslant \tau$. So, even if we have to work with a subsequence of $\left(x_{n}\right)_{n \in \mathbb{N}}$, in (3.1) we can assume that $\delta_{k_{n}} \geqslant \tau$ for every index $n$ and that the sequence $\left(\varphi_{n}\left(x_{n-1}\right)\right)_{n \in \mathbb{N}}$ is convergent with limit $\ell \in \Omega\left(x_{0}\right)$. But reasoning as in the proof of Theorem 2.5, this implies that $A_{i} \ell=0$ and so $\ell \in M_{i}$.

We immediately deduce from Proposition 1.6 and Proposition 3.3 the following corollary.

Corollary 3.4. Suppose that there exists a mode $i$ with $M_{i}=\{0\}$ and which satisfies Assumption 3.1, then $\Omega\left(x_{0}\right)=\{0\}$.

The next proposition tell us that each $\omega$-limit point belongs to at least one set $M_{i}$.

\footnotetext{
${ }^{1} \alpha_{i}(t)=1$ if $t$ belongs to the union of these intervals
} 
Proposition 3.5. We assume that Assumption 3.1 is satisfied by every mode. Then, for every $\ell \in \Omega\left(x_{0}\right)$, there exists a mode $i \in\{1, \ldots, N\}$ such that $\ell \in M_{i}$. In other words, $\Omega\left(x_{0}\right)$ is included in the union $\bigcup_{i=1}^{N} M_{i}$.

Proof. If $\Omega\left(x_{0}\right)$ is a singleton, the result is given by Proposition 3.3. If $\Omega\left(x_{0}\right)$ is not a singleton, let $\ell \in \Omega\left(x_{0}\right)$ and take $\varepsilon>0$ such that $\Omega\left(x_{0}\right)$ contains at least a point outside of the open ball $B(\ell, \varepsilon)$. From the definition of $\Omega\left(x_{0}\right)$, there exists a sequence of times $\left(t_{n}\right)_{n \in \mathbb{N}}$ such that $\lim _{n \rightarrow \infty} x\left(t_{n}\right)=\ell$ and we can assume that $x\left(t_{n}\right) \in B(\ell, \varepsilon)$ for every $n \in \mathbb{N}$. Denote by $t_{n}^{\prime}$ the number defined by $t_{n}^{\prime}=\inf \left\{t>t_{n} \mid x(t) \in \partial B(\ell, \varepsilon)\right\}$. We claim that there exists $\tau>0$ such that, for every $n, t_{n}^{\prime}-t_{n} \geqslant \tau$. The proof of the existence of $\tau$ is by contradiction, suppose that for every $\tau>0$, there exists $n_{0}$ such that $t_{n}^{\prime}-t_{n}<\tau$ as soon as $n>n_{0}$, then there exists an increasing sequence of indices $\left(n_{k}\right)_{k \in \mathbb{N}}$ such that $t_{n_{k}}^{\prime}-t_{n_{k}}<1 / k$. As $x(s)$ is bounded and $t_{n_{k}}^{\prime}-t_{n_{k}}$ tends to 0 , we have

$$
x\left(t_{n_{k}}^{\prime}\right)-x\left(t_{n_{k}}\right)=\int_{t_{n_{k}}}^{t_{n_{k}}^{\prime}} \sum_{i=1}^{N} \alpha_{i}(s) A_{i} x(s) d s \underset{k \rightarrow \infty}{\rightarrow} 0,
$$

which implies that $x\left(t_{n_{k}}^{\prime}\right)$ tends to $\ell$, which contradicts the definition of the sequence $\left(t_{n}^{\prime}\right)_{n \in \mathbb{N}}$. Assume now that for every $i \in\{1, \ldots, N\}$, we have $\left\langle A_{i} \ell, \ell\right\rangle<0$, then there exists $\mu>0$ such that $\left\langle A_{i} \ell, \ell\right\rangle<-\mu$ and, if $\varepsilon$ is chosen small enough, we have $\left\langle A_{i} x(t), x(t)\right\rangle<-\mu / 2$ for every $t$ in the union of intervals $\left[t_{n}, t_{n}^{\prime}\right)$. Hence,

$$
\begin{aligned}
\left\|x\left(t_{n}^{\prime}\right)\right\|^{2}-\left\|x\left(t_{n}\right)\right\|^{2} & =\int_{t_{n}}^{t_{n}^{\prime}} 2 \sum_{i=1}^{N} \alpha_{i}(t)\left\langle A_{i} x(t), x(t)\right\rangle d t \\
& \leqslant-\mu\left(t_{n}^{\prime}-t_{n}\right) \\
& \leqslant-\mu \tau .
\end{aligned}
$$

Even if we have to work with a subsequence of $\left(x\left(t_{n}\right)\right)_{n \in \mathbb{N}}$, we can assume that $t_{n-1}<t_{n-1}^{\prime}<t_{n}$. In this case, $\left\|x\left(t_{k}\right)\right\| \leqslant\left\|x\left(t_{k-1}^{\prime}\right)\right\|$ and we deduce from (3.3) that

$$
\begin{aligned}
\left\|x\left(t_{n}^{\prime}\right)\right\|^{2}-\left\|x\left(t_{0}\right)\right\|^{2} & =\sum_{k=0}^{n}\left(\left\|x\left(t_{k}^{\prime}\right)\right\|^{2}-\left\|x\left(t_{k}\right)\right\|^{2}\right)+\sum_{k=1}^{n}\left(\left\|x\left(t_{k}\right)\right\|^{2}-\left\|x\left(t_{k-1}^{\prime}\right)\right\|^{2}\right) \\
& \leqslant \sum_{k=0}^{n}\left(\left\|x\left(t_{k}^{\prime}\right)\right\|^{2}-\left\|x\left(t_{k}\right)\right\|^{2}\right) \\
& \leqslant-(n+1) \mu \tau
\end{aligned}
$$

which leads to $\left\|x\left(t_{n}^{\prime}\right)\right\|^{2}$ negative if $n$ is chosen large enough which is impossible.

We prove here a proposition announced in the previous section and stating a result of convergence to zero in the two-dimensional case.

Proposition 3.6. In the two-dimensional case $(d=2)$, if every mode of system (1.3) satisfies Assumptions 2.1 and 2.7, then $\Omega\left(x_{0}\right)=\{0\}$ for every $x_{0} \in \mathbb{R}^{2}$.

Proof. Take $x_{0} \in \mathbb{R}^{2}$, the $\omega$-limit set $\Omega\left(x_{0}\right)$ is included in $\bigcup_{i=1}^{N} M_{i}$. Due to Assumption 1.2, the sets $M_{i}$ are zero or one-dimensional subspaces of $\mathbb{R}^{2}$, so their intersection with $S^{1}$ gives a set of isolated points. Assume that $\Omega\left(x_{0}\right)$ is not $\{0\}$, then it is included on a circle with center the origin and radius $r>0$; moreover due to Assumption 3.1, it is also included in the union $\bigcup_{i=1}^{N} M_{i}$. Therefore $\Omega\left(x_{0}\right)$ is included in a finite set of points located on the circle. As $\Omega\left(x_{0}\right)$ 
is a connected set, we deduce that $\Omega\left(x_{0}\right)$ is a single point that we shall denote by $\ell$. Take $i_{0} \in\{1, \ldots, N\}$, due to Assumption 2.7, we can find a sequence $\left(t_{n}\right)_{n \in \mathbb{N}}$ tending to infinity such that we can write $x\left(t_{n}\right)=e^{\tau_{n} A_{i}}\left(x\left(t_{n}-\tau_{n}\right)\right)$ with $\tau_{n}>\tau>0$. Reasoning as in the proof of Theorem 2.5, we deduce that the limit of $x\left(t_{n}-\tau_{n}\right)$ as $n$ tends to infinity belongs to ker $A_{i_{0}}$ but this limit is equal to $\ell$. So we proved that $\ell \in \bigcap_{i=1}^{N} \operatorname{ker} A_{i}$ and as this intersection is $\{0\}$ (Assumption 2.1) we have also $\ell=0$.

In order to give a more precise description of the $\omega$-limit set, we shall assume that the different modes are well distributed. Roughly speaking, this means that the contribution of a given mode cannot be neglected with respect to the contributions of the other modes. Below is the precise definition.

Assumption 3.7 (Persistent excitation). We shall say that the mode $i$ satisfies the "persistent excitation" assumption if, for every sequence of intervals $\left(\left[t_{n}, t_{n}^{\prime}\right]\right)_{n \in \mathbb{N}}$ such that

- the limit of $t_{n}$ as $n$ tends to infinity is equal to infinity;

- there exists $\tau>0$, such that $t_{n}^{\prime}-t_{n} \geqslant \tau$ for every $n \geqslant 0$;

- the limit of the number of commutations occurring in the interval $\left[t_{n}, t_{n}^{\prime}\right]$ tends to infinity as $n$ tends to infinity,

we have $\liminf _{n \rightarrow+\infty} \lambda\left\{t \in\left[t_{n}, t_{n}^{\prime}\right] \mid \alpha_{i}(t)=1\right\}>0$.

Proposition 3.8. Assume that Assumptions 3.1 and 3.7 are satisfied by the mode $i$, then $\Omega\left(x_{0}\right) \subset M_{i}$.

Proof. Let $\ell \in \Omega\left(x_{0}\right)$, if $\Omega\left(x_{0}\right)$ reduces to $\ell$, the result follows from Proposition 3.3; if not, we shall argue by contradiction. So, we suppose that there exists $\ell \in \Omega\left(x_{0}\right)$ such that $\left\langle A_{i} \ell, \ell\right\rangle<-\mu<0$. As in the proof of Proposition 3.5, we take a sequence $\left(t_{n}\right)_{n \in \mathbb{N}}$ such that $\lim _{n \rightarrow+\infty} x\left(t_{n}\right)=\ell$, we choose $\varepsilon>0$ and we define the sequence $\left(t_{n}^{\prime}\right)_{n \in \mathbb{N}}$ by $t_{n}^{\prime}=\inf \left\{t \geqslant t_{n} \mid\right.$ $\left.\left\|x\left(t_{n}^{\prime}\right)-\ell\right\|=\varepsilon\right\}$. The positive number $\varepsilon$ is chosen small enough to have $\left\langle A_{i} x(t), x(t)\right\rangle \leqslant-\mu / 2$ for every $t \in\left[t_{n}, t_{n}^{\prime}\right]$ and, up to a subsequence of $\left(t_{n}^{\prime}\right)_{n \in \mathbb{N}}$, we assume that the limit, denoted by $\ell^{\prime}$, of the sequence $\left(x\left(t_{n}^{\prime}\right)\right)_{n \geqslant 1}$ exists. As in the proof of Proposition 3.5, we can show that there exists $\tau>0$ with $t_{n}^{\prime}-t_{n} \geqslant \tau$.

Moreover, the number of commutations occurring in the interval $\left[t_{n}, t_{n}^{\prime}\right]$ cannot be bounded. If it were the case, up to a subsequence of $\left(\left[t_{n}, t_{n}^{\prime}\right]\right)_{n \in \mathbb{N}}$, we could assume that there exists a finite sequence $\left(i_{1}, \ldots, i_{r}\right)$ of indices taken in $\{1, \ldots, N\}$ such that $x\left(t_{n}^{\prime}\right)=e^{u_{n}^{1} A_{i_{1}}} \circ \cdots \circ$ $e^{u_{n}^{r} A_{i_{r}}} x\left(t_{n}\right)$, with $u_{n}^{1}, \ldots, u_{n}^{r} \geqslant 0$. As in the proof of Theorem 2.5, in this case, we could show that $\lim _{n \rightarrow+\infty} x\left(t_{n}^{\prime}\right)=\ell$ which is impossible since $\left\|\ell^{\prime}-\ell\right\|=\varepsilon$. So, we can suppose that the limit, as $n$ tends to infinity, of the number of commutations occurring in the interval $\left[t_{n}, t_{n}^{\prime}\right]$ is infinite. Therefore, denting by $J_{n}$ the set $J_{n}=\left\{t \in\left[t_{n}, t_{n}^{\prime}\right] \mid \alpha_{i}(t)=1\right\}$, from Assumption 3.7, we have $\liminf _{n \rightarrow+\infty} \lambda\left(J_{n}\right)=\tau_{i}>0$. Now we have

$$
\begin{aligned}
\left\|x\left(t_{n}^{\prime}\right)\right\|^{2}-\left\|x\left(t_{n}\right)\right\|^{2} & =2 \int_{t_{n}}^{t_{n}^{\prime}} \sum_{j=1}^{N} \alpha_{j}(s)\left\langle A_{j} x(s), x(s)\right\rangle d t \\
& \leqslant 2 \int_{J_{n}}\left\langle A_{i} x(s), x(s)\right\rangle d t \\
& \leqslant-\mu \lambda\left(J_{n}\right) .
\end{aligned}
$$


But $\lambda\left(J_{n}\right) \geqslant \tau_{i} / 2$ if $n$ is large enough and so from (3.4), we get

$$
\left\|x\left(t_{n}^{\prime}\right)\right\|^{2}-\left\|x\left(t_{n}\right)\right\|^{2} \leqslant-\frac{\tau_{i} \mu}{2}
$$

for $n$ large enough. Passing to the limit in (3.5), we get $\left\|\ell^{\prime}\right\|^{2}-\|\ell\|^{2} \leqslant-\frac{\tau_{i} \mu}{2}$ which is impossible since $\left\|\ell^{\prime}\right\|=\|\ell\|$.

From Proposition 3.8 we deduce the following easy consequence.

Corollary 3.9. If Assumption 3.7 is satisfied and if $M=\{0\}$, then $x(t)$, the solution of system (1.3), tends to zero as t tends to infinity.

Proof. If Assumption 3.7 is satisfied, then $\Omega\left(x_{0}\right)$ reduces to $\{0\}$.

Remark. We may wonder if we could weaken the hypothesis in Proposition 3.8 by assuming only the permanent excitation (Assumption 3.1). The following example gives a negative answer to this question.

Example 3.10. In this example, we take the matrices $A_{1}$ and $A_{2}$ from Example 2.2 as well as the following matrix $A_{3}$ defined as

$$
A_{3}=\left(\begin{array}{ccc}
-1 & 0 & 0 \\
0 & 0 & 0 \\
0 & 0 & 0
\end{array}\right)
$$

Put $\psi_{n}=\left(e^{t_{0} A_{1} / n} \circ e^{t_{0} A_{2} / n} \circ e^{t_{0} A_{3} / n^{2}}\right)^{n}$ with $t_{0}=\pi / 4$. We have $\lim _{n \rightarrow+\infty} \psi_{n}=e^{t_{0}\left(A_{1}+A_{2}\right)}$. Given integers $n_{1}, \ldots, n_{4}$, set

$$
\Theta\left(n_{1}, n_{2}, n_{3}, n_{4}\right)=\psi_{n_{4}} \circ e^{t_{0} A_{3}} \circ \psi_{n_{3}} \circ \psi_{n_{2}} \circ e^{t_{0} A_{3}} \circ \psi_{n_{1}} .
$$

A positive number $\varepsilon$ being given, proceeding as in Example 2.2, one can prove that it is possible to choose the integers $n_{1}, \ldots, n_{4}$ in such a way that $\left\|\Theta\left(n_{1}, n_{2}, n_{3}, n_{4}\right)\left(e_{0}\right)-e_{0}\right\| \leqslant \varepsilon / 2$; more generally, we can find a sequence $\left(n_{k}\right)_{k \in \mathbb{N}}$ such that

$$
\left\|\Theta\left(n_{4 k+1}, n_{4 k+2}, n_{4 k+3}, n_{4 k}\right) \circ \cdots \circ \Theta\left(n_{1}, n_{2}, n_{3}, n_{4}\right)\left(e_{0}\right)-e_{0}\right\| \leqslant \frac{\varepsilon}{2}+\cdots+\frac{\varepsilon}{2^{k+1}} .
$$

We choose now a switch law such that the solution of (1.3) (with $x_{0}=e_{0}$ ) is such that

$$
x\left(6(k+1) t_{0}+\sum_{i=1}^{4 k+4} t_{0} / n_{i}\right)=\Theta\left(n_{4 k+1}, n_{4 k+2}, n_{4 k+3}, n_{4 k}\right) \circ \cdots \circ \Theta\left(n_{1}, n_{2}, n_{3}, n_{4}\right)\left(e_{0}\right) .
$$

For this switch law, inequality (3.6) shows that there exists an $\omega$-limit point $\ell$ in the open ball $B\left(e_{0}, \varepsilon\right)$ and, if $\varepsilon$ is chosen small enough this limit point is such that $\left\langle A_{3} \ell, \ell\right\rangle \neq 0$.

\subsection{What happens when $\Omega\left(x_{0}\right)$ is a singleton}

In this section, we freely use the convenient chronological calculus notation which allows an exponentiel representation of flows and for which we refer to [1, Chapter 2].

The following result is well-known, but for the convenience of the reader we shall supply a simple proof.

Lemma 3.11. Let $\left(\varphi_{n}\right)_{n \in \mathbb{N}}$ be a sequence in $\boldsymbol{L}^{\infty}\left(\mathbb{R}_{+}, \mathcal{B}\right)$ where $\mathcal{B}$ is a bounded subset of $\mathbb{R}^{N}$. If $\varphi_{n} \stackrel{*}{\rightarrow} \varphi$, then $\varphi$ takes values in $\overline{\mathrm{co}}(\mathcal{B})$ (the closed convex hull of $\mathcal{B}$ ) for almost every $t$ in $\mathbb{R}_{+}$. 
Proof. Let $\mathcal{L}$ denote the set of affine forms of $\mathbb{R}^{N}$ with rational coefficients. Let $\mathcal{L}^{\mathcal{B}}=\{L \in$ $\left.\mathcal{L} \mid L(\mathcal{B}) \subset \mathbb{R}_{+}\right\}$. We have $\overline{\mathrm{co}}(\mathcal{B})=\bigcap_{L \in \mathcal{L}^{\mathcal{B}}} L^{-1}\left(\mathbb{R}_{+}\right)$. Take $L \in \mathcal{L}^{\mathcal{B}}$. Since $\varphi_{n}$ takes values in $\mathcal{B}$, $\int_{A} L\left(\varphi_{n}(t)\right) d t \geqslant 0$, for any measurable subset $A \subset \mathbb{R}_{+}$. Since $\varphi_{n} \stackrel{*}{\rightarrow} \varphi$ and $L$ is continuous, we get for any measurable set $A \subset \mathbb{R}_{+}$

$$
\int_{A} L(\varphi(t)) d t=L\left(\int_{A} \varphi(t) d t\right)=\lim _{n \rightarrow+\infty} L\left(\int_{A} \varphi_{n}(t) d t\right)=\lim _{n \rightarrow+\infty} \int_{A} L\left(\varphi_{n}(t)\right) d t \geqslant 0,
$$

which implies that $L \circ \varphi$ is almost surely nonnegative on $\mathbb{R}_{+}$. In other words, $\lambda\{t \geqslant 0 \mid$ $L \circ \varphi(t)<0\}=0$ for every $L \in \mathcal{L}^{\mathcal{B}}$. Using the countability of $\mathcal{L}^{\mathcal{B}}$, it follows that

$$
\begin{aligned}
\lambda(\{t \geqslant 0 \mid \varphi(t) \notin \overline{\mathrm{co}}(\mathcal{B})\}) & =\lambda\left(\left\{\cup_{L \in \mathcal{L}^{\mathcal{B}}}(L \circ \varphi)^{-1}((-\infty, 0))\right\}\right) \\
& \leqslant \sum_{L \in \mathcal{L}^{\mathcal{B}}} \lambda(\{t \geqslant 0 \mid L \circ \varphi(t)<0\})=0,
\end{aligned}
$$

or, equivalently that $\varphi(t) \in \overline{\mathrm{co}}(\mathcal{B})$ almost surely.

We denote by $\Delta_{\geqslant 0}$ and $\Delta_{>0}$ the sets defined by

$$
\Delta_{\geqslant 0}=\left\{\alpha \in \mathbb{R}_{+}^{N} \mid \alpha_{1}+\cdots+\alpha_{N}=1\right\},
$$

and

$$
\Delta_{>0}=\left\{\alpha \in \Delta_{\geqslant 0} \mid \alpha_{i}>0, \forall i=1, \ldots, N\right\} .
$$

As consequence of Lemma 3.11 to switched linear system we have the following results. We begin with proving the following proposition.

Proposition 3.12. If $\Omega\left(x_{0}\right)=\ell$, then there exists $\alpha \in \Delta_{\geqslant 0}$ such that $\sum_{i=1}^{N} \alpha_{i} A_{i} \ell=0$.

Proof. Let $\mathcal{B}=\left\{e_{1}, \ldots, e_{N}\right\}$ be the canonical basis of $\mathbb{R}^{N}$. Let $\left(t_{n}\right)_{n \in \mathbb{N}}$ be a sequence of positive numbers tending to infinity. Define $\beta \in \boldsymbol{L}^{\infty}\left(\mathbb{R}_{+}, \mathcal{B}\right)$ by $\beta(t)=e_{i}$ if $\alpha(t)=i$. Set $\varphi_{n}(t)=$ $\left.\beta\right|_{\left[t_{n}, t_{n+1}\right]}(n+t)$. For all $n \geqslant 1$, we have $\varphi_{n} \in \boldsymbol{L}^{\infty}\left(\mathbb{R}_{+}, \mathcal{B}\right)$. It follows from Alaoglu's theorem (see e.g. [7, Theorem III.15]) and Lemma 3.11 that $\varphi_{n}$ admits a converging subsequense $\varphi_{n_{k}} \stackrel{*}{\rightarrow} \varphi \in \boldsymbol{L}^{\infty}\left(\mathbb{R}_{+}, \overline{\mathrm{CO}}(\mathcal{B})\right)$. Thus, $\varphi(t)=\sum_{k=1}^{N} \alpha_{i}(t) e_{i}$, where the $\alpha_{i}(\cdot)$ 's are non negative measurable functions. To each $\varphi_{n_{k}}$ corresponds a nonautonomus vector field $X_{t}^{n_{k}}$ defined by

$$
X_{t}^{n_{k}}(x)=\sum_{i=1}^{N}\left\langle\varphi_{n_{k}}(t), e_{i}\right\rangle A_{i} x .
$$

Consequently, for every $x \in \mathbb{R}^{d}, X^{n_{k}}(x) \underset{k \rightarrow \infty}{\stackrel{*}{*}} X .(x)=\sum_{i=1}^{N} \alpha_{i}(\cdot) A_{i} x$, which implies in particular, that for every $t \geqslant 0$,

$$
\int_{0}^{t} X_{s}^{n_{k}} d s \underset{k \rightarrow \infty}{\rightarrow} \int_{0}^{t} X_{s} d s
$$

It is easy to see that this convergence is indeed uniform with respect to $(t, x)$ on every compact subset of $\mathbb{R}_{+} \times \mathbb{R}^{d}$ (we prove this fact in Lemma 3.13 after the present proof), and because the $X_{t}^{n_{k}}$ are linear (in $x$ ), the same property of uniform convergence holds for all derivatives with respect to $x$. Hence (see [1, Lemma 8.10]),

$$
\overrightarrow{\exp } \int_{0}^{t} X_{s}^{n_{k}} d s \underset{k \rightarrow \infty}{\rightarrow} \overrightarrow{\exp } \int_{0}^{t} X_{s} d s,^{2}
$$

\footnotetext{
${ }^{2}$ If $Y_{t}$ is a nonautonomous vector field, $\overrightarrow{\exp } \int_{0}^{t} Y_{s} d s$ stands for its flow (for details on this notation see [1, Chapter 2]).
} 
uniformly with respect to $(t, x)$ on every compact subset of $\mathbb{R}_{+} \times \mathbb{R}^{d}$. In particular, it is true for the compact $\mathcal{K}=[0, t] \times \overline{\left\{x(s) \mid s \in \mathbb{R}_{+}\right\}}$. In other words,

$$
\forall \varepsilon>0, \quad \exists k_{0} \in \mathbb{N} \mid\left\|\overrightarrow{\exp } \int_{0}^{t} X_{s} d s(z)-\overrightarrow{\exp } \int_{0}^{t} X_{s}^{n_{k}} d s(z)\right\|<\varepsilon,
$$

for all $k \geqslant k_{0}$ and all $z \in \mathcal{K}$. In particular, for $z=x\left(t_{n_{k}}\right)$,

$$
\begin{aligned}
& \left\|\overrightarrow{\exp } \int_{0}^{t} X_{s} d s\left(x\left(t_{n_{k}}\right)\right)-\overrightarrow{\exp } \int_{0}^{t} X_{s}^{n_{k}} d s\left(x\left(t_{n_{k}}\right)\right)\right\| \\
& =\left\|\overrightarrow{\exp } \int_{0}^{t} X_{s} d s\left(x\left(t_{n_{k}}\right)\right)-x\left(t_{n_{k}}+t\right)\right\|<\varepsilon .
\end{aligned}
$$

and passing to the limit as $k$ goes to infinity in the previous equation, we get that for every $\varepsilon$, $t>0,\left\|\overrightarrow{\exp } \int_{0}^{t} X_{s} d s(\ell)-\ell\right\|<\varepsilon$, or equivalently that, for every $t \geqslant 0, \overrightarrow{\exp } \int_{0}^{t} X_{s} d s(\ell)=\ell$, from which follows the result after differentiating with respect to $t$.

Lemma 3.13. The convergence in relation (3.8) is uniform with respect to $(t, x)$ on every compact subset of $\mathbb{R}_{+} \times \mathbb{R}^{d}$.

Proof. In view of relation (3.7), and because the mappings $x \mapsto A_{i} x$ are linear, it is sufficient to show that the convergence (which holds true since $\varphi_{n_{k}} \stackrel{*}{\rightarrow} \varphi$ )

$$
\int_{0}^{T} \varphi_{n_{k}}(s) d s \underset{k \rightarrow \infty}{\rightarrow} \int_{0}^{T} \varphi(s) d s
$$

is uniform with respect to $t$ on $[0, T]$. Fix $\varepsilon>0$ and $p \in \mathbb{N}$ such that $\frac{5}{2^{p}} \leqslant \varepsilon$. Set $I_{p}^{q}=$ $\left[\frac{q}{p} T, \frac{q+1}{p} T\left[\right.\right.$. Since $\varphi_{n_{k}} \stackrel{*}{\rightarrow} \varphi$, there exists $k_{0}(p, q)$ such that

$$
\left|\int_{0}^{T} \chi_{I_{p}^{q}}(s)\left(\varphi_{n_{p}}-\varphi\right)(s) d s\right| \leqslant \frac{1}{k 2^{i+1}}, \quad \forall k \geqslant k_{0}(p, q) .
$$

Let $k_{0}=\max \left\{k_{0}(p, 1), \ldots, k_{0}(p, p)\right\}$. Denote by $q_{t}$ the index for which $t \in I_{p}^{q_{t}}$. For all $k \geqslant k_{0}$, we have,

$$
\begin{aligned}
\left|\int_{0}^{t}\left(\varphi_{n_{k}}-\varphi\right)(s) d s\right| & \leqslant \sum_{q=0}^{p-1}\left|\int_{0}^{t} \chi_{I_{p}^{q}}(s)\left(\varphi_{n_{k}}-\varphi\right)(s) d s\right| \\
& =\sum_{q \neq q_{t}}\left|\int_{0}^{T} \chi_{I_{p}^{q}}(s)\left(\varphi_{n_{k}}-\varphi\right)(s) d s\right|+\left|\int_{0}^{t}\left(\varphi_{n_{k}}-\varphi\right)(s) d s\right| \\
& \leqslant \sum_{q=0}^{p-1}\left|\int_{0}^{T} \chi_{I_{p}^{q}}(s)\left(\varphi_{n_{k}}-\varphi\right)(s) d s\right|+\frac{2}{p}\left\|\varphi_{n_{k}}-\varphi\right\|_{\infty} \leqslant \varepsilon
\end{aligned}
$$

from which the uniform convergence (3.8) follows.

Definition 3.14. We say that a subset $I \in\{1, \ldots, N\}$ is minimal (with respect to $\ell$ ) if there exists a unique $\alpha=\left(\alpha_{1}^{0}, \ldots, \alpha_{n}^{0}\right) \in \Delta_{>0}$ such that $\sum_{i \in I} \alpha_{i}^{0} A_{i} \ell=0$.

We now prove that Assumption 3.7 (and thus Assumption 3.1) is necessary under the STRONG condition of minimality (whose definition is below) of the set $\{1, \ldots, N\}$. 
Theorem 3.15. Assume that $\Omega\left(x_{0}\right)=\{\ell\}$. If $\{1, \ldots, N\}$ is minimal (with respect to $\ell$ ), then Assumption 3.7 is satisfied.

Proof. Let $\alpha^{0}=\left(\alpha_{1}^{0}, \ldots, \alpha_{N}^{0}\right)$ be the unique element of $\Delta_{>0}$ such that $\sum_{i=1}^{N} \alpha_{i}^{0} A_{i} \ell=0$. According to Lemma 3.12, there exists $\alpha \in \Delta_{\geqslant 0}$ such that $\sum_{i=1}^{N} \alpha_{i} A_{i} \ell=0$. It follows from the minimality of $\{1, \ldots, N\}$ that $\alpha=\alpha^{0}$. In particular, $\alpha_{i}>0$ for all $i \in\{1, \ldots, N\}$. Keeping the same notation as in the proof of Lemma 3.12, we thus have proved that all the (weak star) convergent subsequences of $\varphi_{n}$ converge to $\sum_{i=1}^{N} \alpha_{i}^{0} e_{i}$. Consequently, $\varphi_{n}$ converges to $\sum_{i=1}^{N} \alpha_{i}^{0} e_{i}$. In particular, $\left\langle\varphi_{n}, e_{i}\right\rangle \stackrel{*}{\rightarrow} \alpha_{i}^{0}$ for all $i \in\{1, \ldots, N\}$. Consequently, taking $t_{n}=n$, we have,

$$
\lambda\left(\left\{t \in[n, n+1] \mid \alpha_{i}(t)=1\right\}\right)=\int_{n}^{n+1}\left\langle\beta(s), e_{i}\right\rangle d s=\int_{0}^{1}\left\langle\varphi_{n}, e_{i}\right\rangle \rightarrow \alpha_{i}^{0}>0 .
$$

This computation proves that the total time of activation of the mode $i$ on an interval $\left[t_{n}, t_{n}^{\prime}\right]$ (where $\lim _{n \rightarrow+\infty} t_{n}=+\infty$ ) is equivalent, as $n \rightarrow+\infty$, to $\alpha_{i}^{0}\left(t_{n}^{\prime}-t_{n}\right)$, consequently, Assumption 3.7 is satisfied.

\subsection{Further remarks}

In subsection 3.1, we have seen that, under Assumptions 3.1, the set $\Omega\left(x_{0}\right)$ is included in $\bigcup_{i=1}^{N} M_{i}$; moreover in subsection 3.2, we have seen that, in the case where $\Omega\left(x_{0}\right)$ reduces to an unique point $\ell$, there exists a convex combination of the vectors $A_{i} \ell$ which vanishes. In this subsection, we shall see what we can say of the convex combinations of the $A_{i} \ell^{\prime}$ s in the general case. We begin with a definition.

Definition 3.16. We shall say that $\ell$ is an ordinary point of $\bigcup_{i=1}^{N} M_{i}$ if, whenever we have $i_{1}$ and $i_{2}$ in $I_{\ell}$, either $M_{i_{1}} \subset M_{i_{2}}$ or $M_{i_{2}} \subset M_{i_{1}}$. We shall say that $\ell \in \bigcup_{i=1}^{N} M_{i}$ is an extraordinary point if it is not ordinary.

Notice that if $\ell \in \bigcup_{i=1}^{N} M_{i}$ is an ordinary point, there exists an index $i_{\ell}$ such that $\bigcup_{i \in I_{\ell}} M_{i}=$ $M_{i_{\ell}}$. Clearly, the set of ordinary points of $\bigcup_{i=1}^{N} M_{i}$ is an open and dense subset of $\bigcup_{i=1}^{N} M_{i}$. Moreover, if $\ell$ is an ordinary point of $\bigcup_{i=1}^{N} M_{i}$, there exists an open neighborhood $U$ of $\ell$ such that $U \cap \bigcup_{i=1}^{N} M_{i}=U \cap \bigcup_{i \in I_{\ell}} M_{i}$. The following proposition gives an additional condition to be satisfied by a point $\ell$ in order it belongs to $\Omega\left(x_{0}\right)$.

Proposition 3.17. Assume that $\ell \in \Omega\left(x_{0}\right)$. There exists $\alpha \in \Delta_{\geqslant 0}$ such that

1. if $\ell$ is an ordinary point, $\sum_{i=1}^{N} \alpha_{i} A_{i} \ell \in \bigcup_{i \in I_{\ell}} M_{i}$,

2. if $\ell$ is an extraordinary point, there exists $I_{\ell}^{\prime} \subset I_{\ell}$ such that $\sum_{i=1}^{N} \alpha_{i} A_{i} \ell \in \bigcap_{i \in I_{\ell}^{\prime}} M_{i}$.

Proof. Assume first that $\ell$ is an ordinary point of $\Omega\left(x_{0}\right)$. Assume that, for every $\alpha \in \Delta_{\geqslant 0}$, the sum $\sum_{i=1}^{N} \alpha_{i} A_{i} \ell$ does not belong to $\bigcup_{i \in I_{\ell}} M_{i}$, then there exists a vector $u$ orthogonal to $\bigcup_{i \in I_{\ell}} M_{i}=M_{i_{\ell}}$ and a positive constant $c$, such that $\left\langle A_{i} \ell, u\right\rangle>c>0$ for every $i \in I_{\ell}$. Choose $U=B(\ell, r)$ a small enough open ball around $\ell$ such that $U \cap \bigcup_{i=1}^{N} M_{i}=U \cap \bigcup_{i \in I_{\ell}} M_{i}$ and $\left\langle A_{i} \ell^{\prime}, u\right\rangle \geqslant c / 2$ for every $\ell^{\prime} \in U \cap \bigcup_{i \in I_{\ell}} M_{i}$. There exists a sequence $\left(t_{n}\right)_{n \in \mathbb{N}}$ tending to infinity such that $\lim _{n \rightarrow+\infty} x\left(t_{n}\right)=\ell$ and $x\left(t_{n}\right) \in B(\ell, r / 2)$ for every $n \in \mathbb{N}$.

If $\Omega\left(x_{0}\right)$ reduces to $\ell$, put $\tau_{n}=1$. Since $\lim _{n \rightarrow+\infty} x\left(t_{n}+\tau_{n}\right)=\ell, x\left(t_{n}+\tau_{n}\right) \in B(\ell, r / 2)$ if the index $n$ is large enough. If $\Omega\left(x_{0}\right)$ does not reduce to $\ell$, there exists an $\omega$-limit point outside of the ball $B(\ell, r)$ if $r$ is chosen small enough. In this last case, we choose $\tau_{n}=\inf \{t>$ 
$\left.t_{n} \mid x(t) \in \partial B(\ell, r / 2)\right\}$ which is well defined for every index $n$. In any case, the sequence $\left(x\left(t_{n}+\tau_{n}\right)\right)_{n \in \mathbb{N}}$ being bounded, we can assume that it converges to $\bar{\ell} \in U$. We have

$$
\left\langle x\left(t_{n}+\tau_{n}\right)-x\left(t_{n}\right), u\right\rangle=\int_{t_{n}}^{t_{n}+\tau_{n}} \sum_{i=1}^{N} \alpha_{i}(s)\left\langle A_{i} x(s), u\right\rangle d s \geqslant \frac{c}{2} \tau_{n},
$$

if $n$ is large enough. If $\ell=\bar{\ell}$, put $\tau_{n}=1$ for every index $n$. If $\bar{\ell} \neq \ell$, as we cannot have $\liminf \operatorname{in}_{n \rightarrow+\infty} \tau_{n}=0$, there exists $\tau>0$ such that $\tau_{n}>\tau$ as soon as index $n$ is large enough, so from (3.12), we deduce

$$
\frac{c}{2} \tau \leqslant\left\langle x\left(t_{n}+\tau_{n}\right)-x\left(t_{n}\right), u\right\rangle .
$$

As $\bar{\ell} \in \bigcup_{i \in I_{\ell}} M_{i}$, the limit of the right-hand side of (3.13) is 0 , which leads to a contradiction.

Assume now that $\ell$ is an extraordinary point in $\bigcup_{i=1}^{N} M_{i}$, if $\ell$ is the limit of a sequence $\left(\ell_{k}\right)_{k \in \mathbb{N}}$ of ordinary points, we can assume that the sets of indices $I_{\ell_{k}}$ are all equal to a set $I_{0} \subset\{1, \ldots, N\}$. So, there exists a sequence $\left(\alpha^{\ell_{k}}\right)_{k \in \mathbb{N}}$ of elements of $\Delta_{\geqslant 0}$ such that

$$
\sum_{i=1}^{N} \alpha_{i}^{\ell_{k}} A_{i} \ell_{k} \in \bigcup_{i \in I_{0}} M_{i}
$$

Now, as $\Delta_{\geqslant 0}$ is compact, we can suppose that the sequence $\left(\alpha^{\ell_{k}}\right)_{k \in \mathbb{N}}$ converges to $\alpha \in \Delta_{\geqslant 0}$ and equality (3.14) implies $\sum_{i=1}^{N} \alpha_{i} A_{i} \ell \in \bigcup_{i \in I_{0}} M_{i}$. If there exists an open neighborhood $U$ of $\ell$ such that $U \cap \bigcup_{i=1}^{N} M_{i}$ is constituted by extraordinary points, let $\left(\ell_{k}\right)_{k \in \mathbb{N}}$ be a sequence of extraordinary points tending to $\ell$, we can assume that all the subsets of indices $I_{\ell_{k}}$ are equal to $I_{0}$ and we can also assume that all the $\ell_{k}$ as well as $\ell$ belong to a same intersection of subspaces $\bigcap_{i \in I_{0}} M_{i}$, reasoning as in the first part of this proof, we get the result stated in the proposition.

\subsection{Nonconvergence to zero of the switched system under some weak hy- pothesis}

The next two results show that Assumption 3.7 is not sufficient to ensure the converge to zero of system (1.3) when the space $M$ of non detectability is not reduced to zero.

Proposition 3.18. Let $\ell \in M \backslash\{0\}$. Suppose that there exists $\alpha=\left(\alpha_{1}, \cdots, \alpha_{N}\right) \in \Delta_{>0}$ such that $\sum_{i=1}^{N} \alpha_{i} A_{i} \ell=0$. Then, there exists a trajectory satisfying Assumption 3.7 which does not converge to zero.

Proof. Let $\left(\alpha_{1}, \ldots, \alpha_{N}\right) \in \Delta_{>0}$ be such that $\sum_{i=1}^{N} \alpha_{i} A_{i} \ell=0$. For any fixed $t \in[0,1]$ define the linear map $\Phi^{t}=e^{t \alpha_{N} A_{N}} \circ \cdots \circ e^{t \alpha_{1} A_{1}}$. The mapping $t \mapsto \Phi^{t}(\ell)$ has the Taylor expansion

$$
\Phi^{t}(\ell)=\Phi^{0}(\ell)+\left.t \frac{d}{d t} \Phi^{t}(\ell)\right|_{t=0}+t^{2} v_{t}=\ell+t \sum_{i=1}^{N} \alpha_{i} A_{i} \ell+t^{2} v_{t}=\ell+t^{2} v_{t}
$$

where the vector $v_{t}$ satisfies $v_{t}=O(1)$ since $[0,1]$ is a compact interval. The vector $v_{t}$ is thus bounded, i.e., there exists $v_{0}$ such that $\left\|v_{t}\right\| \leqslant\left\|v_{0}\right\|$ for all $t \in[0,1]$, which implies that $\left\|\Phi^{t}(\ell)-\ell\right\| \leqslant t^{2}\left\|v_{0}\right\|$. Let $\left(t_{n}\right)_{n \in \mathbb{N}}$ be a sequence in $[0,1]$. We have

$$
\Phi^{t_{n+1}} \circ \Phi^{t_{n}}(\ell)=\Phi^{t_{n+1}}\left(\ell+t_{n}^{2} v_{t_{n}}\right)=\ell+t_{n+1}^{2} v_{t_{n+1}}+t_{n}^{2} \Phi^{t_{n+1}}\left(v_{t_{n}}\right) .
$$

Since $\left\|\Phi^{t}(v)\right\| \leqslant\|v\|$, we have

$$
\left\|\Phi^{t_{n+1}} \circ \Phi^{t_{n}}(\ell)-\ell\right\| \leqslant t_{n+1}^{2}\left\|v_{t_{n+1}}\right\|+t_{n}^{2}\left\|\Phi^{t_{n+1}}\left(v_{t_{n}}\right)\right\| \leqslant\left(t_{n+1}^{2}+t_{n}^{2}\right)\left\|v_{0}\right\| .
$$


Put $\Phi_{p, q}=\Phi^{t_{q}} \circ \cdots \circ \Phi^{t_{p}}$. By induction, we get, for $q \geqslant p,\left\|\Phi_{p, q}(\ell)-\ell\right\| \leqslant\left\|v_{0}\right\| \sum_{n=p}^{q} t_{n}^{2}$, and letting $q$ go to infinity leads to $\lim _{q \rightarrow+\infty}\left\|\Phi_{p, q}(\ell)-\ell\right\| \leqslant\left\|v_{0}\right\| \sum_{n=p}^{\infty} t_{n}^{2}$. As the $\Phi_{p, q}$ 's are equibounded, we can select a converging subsequence $\Phi_{p, q_{k}}$. Set $\Psi_{p}=\lim _{k \rightarrow+\infty} \Phi_{p, q_{k}}$. Now, taking (for example) $t_{n}=\frac{1}{n}$ and $p$ sufficiently large, the above inequality implies that

$$
\forall \varepsilon>0 \quad \exists p_{\varepsilon} \quad \mid \quad \forall p \geqslant p_{\varepsilon} \quad\left\|\Psi_{p}(\ell)-\ell\right\| \leqslant \varepsilon,
$$

which shows in particular that $\Psi_{p}(\ell) \neq 0$. Moreover, because $\alpha \in \Delta_{>0}$, the constructed trajectory satisfies Assumption 3.7.

Proposition 3.19. Suppose that there exists $\alpha=\left(\alpha_{1}, \cdots, \alpha_{N}\right) \in \Delta_{>0}$ such that $M \subset$ $\operatorname{ker} \sum_{i=1}^{N} \alpha_{i} A_{i}$. Then, for every $\ell \in M$, there exists a trajectory, satisfying the permanent excitation and the persistent excitation assumptions, and such that $\ell \in \Omega\left(x_{0}\right)$.

Proof. Let $\left(\alpha_{1}, \ldots, \alpha_{N}\right) \in \Delta_{>0}$ be such that $M \subset \operatorname{ker} \sum_{i=1}^{N} \alpha_{i} A_{i}$ and let $\Psi_{p}$ be defined as in the proof of Theorem 3.18 according to which it remains to show that for any point in $M$, there exists a trajectory that converges to this point. Because $M \subset \operatorname{ker} \sum_{i=1}^{N} \alpha_{i} A_{i}$, relation (3.15) can be rewritten as

$$
\forall \ell \in M \quad \forall \varepsilon>0 \quad \exists p_{\varepsilon} \quad \mid \quad \forall p \geqslant p_{\varepsilon} \quad\left\|\Psi_{p}(\ell)-\ell\right\| \leqslant \varepsilon .
$$

To get the result it is sufficient to show that there exists a $p$ such that the image by $\Psi_{p}$ of an open ball in $M$ centered at the origin contains an open ball. Let $S_{M}$ be the unit sphere of $M$ that is the boundary of the open unit ball $B_{M}$. Let $0<\varepsilon<1$. Since all mappings $\Phi^{t}$ are Lipschitzian with constant one, all mappings $\Psi_{p}$ Lipschitzian with constant one. Thus the family $\left(\Psi_{p}\right)_{p \geqslant 0}$ is equicontinuous. Relation (3.16) indicates that as $p$ goes to infinity, $\left.\Psi_{p} \rightarrow \mathrm{Id}\right|_{M}$, pointwise, thus uniformly. Consequently, there exists $p_{0}$ such that $\left\|\Psi_{p_{0}}(\ell)-\ell\right\|<\varepsilon$ holds for all $\ell \in S_{M}$ from which it follows that $B_{M}(0,1-\varepsilon) \subset \Psi_{p}\left(B_{M}\right)$ (see [22, Lemma 7.23]). This completes the proof.

\section{Application to the three-cell converter}

In this section we apply our theoretical result to the (non linear) continuous-time model of the multilevel converters. For simplicity, we limit ourselves to the case of the three-cell converter although all our results are true for $p$-cell converters with $p \geqslant 3$. Some particularity of the two-cell converter which follows from Proposition 3.6 will be explained at the end of present section.

\subsection{Description of the system}

The circuit topology of the three-cell converter is represented in the picture below.

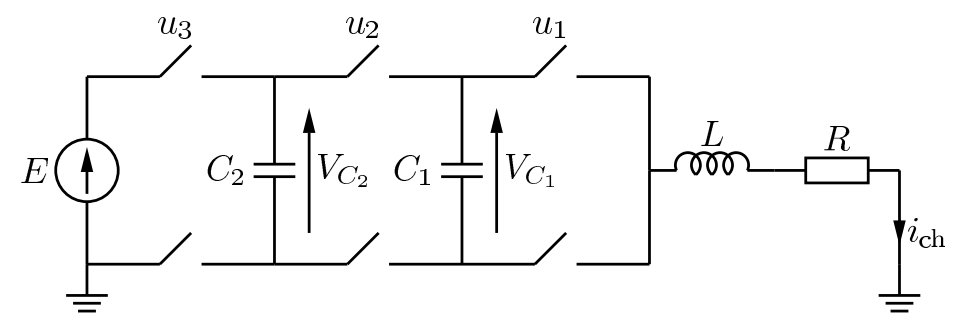

Figure 1: Sketch of the three-cell converter 
Our main goal is to estimate the voltage of the capacitors in the case where only the current in the load is measured. Defining $x(t)=\left(x_{1}(t), x_{2}(t), x_{3}(t)\right)=\left(V_{C_{1}}, V_{C_{2}}, i_{c h}\right)$ as the state vector, where $V_{C_{1}}, V_{C_{2}}$ are the voltages of the corresponding capacitors, $i_{c h}$ is the load current and $y=i_{c h}$ is the output, the model can be represented by a unique state equation:

$$
\left\{\begin{array}{l}
\dot{x}=F(u) x+E G(u) \\
y=C x
\end{array}\right.
$$

where $E$ is the input voltage, $u=\left(u_{1}, u_{2}, u_{3}\right) \in\{0,1\}^{3}$ is the control vector and the matrices $F(u), G(u)$ and $C$ are given by

$$
F(u)=\left(\begin{array}{ccc}
0 & 0 & \frac{u_{2}-u_{1}}{C_{1}} \\
0 & 0 & \frac{u_{3}-u_{2}}{C_{2}} \\
-\frac{u_{2}-u_{1}}{L} & -\frac{u_{3}-u_{2}}{L} & -\frac{R}{L}
\end{array}\right), \quad G(u)=\left(\begin{array}{c}
0 \\
0 \\
\frac{u_{3}}{L}
\end{array}\right), \quad C^{\mathrm{T}}=\left(\begin{array}{l}
0 \\
0 \\
1
\end{array}\right) .
$$

In order to achieve our goal, we build a Luenberger switched observer based on the load current measurement (other approaches are possible see for instance $[3,4,5]$ ). Such an observer takes the form

$$
\dot{\hat{x}}=F(u) \hat{x}-L(u)(C \hat{x}-y)+G(u), \quad L(u) \in \mathbb{R}^{3 \times 1},
$$

and the dynamics of the error $e=\hat{x}-x$ reads

$$
\dot{e}(t)=A(u) e(t), \quad A(u)=F(u)-L(u) C .
$$

Using the convention $i=\sum_{j=1}^{3} u_{j} 2^{j-1}$, we can rewrite this last equation as

$$
\dot{x}(t)=\sum_{i=1}^{8} \alpha_{i}(t) A_{i} x(t), \quad \alpha_{i}(t) \in\{0,1\}, \quad \sum_{i=1}^{8} \alpha_{i}(t)=1 .
$$

It is easy to show that the gain matrices $L_{i}$ can be chosen in such a way that family $\left\{A_{1}, \ldots, A_{8}\right\}$ satisfies Assumption 1.2 Straightforward calculations show that necessarily,

$$
P=\left(\begin{array}{ccc}
p_{11} & p_{12} & 0 \\
p_{12} & p_{22} & 0 \\
0 & 0 & p_{33}
\end{array}\right), \quad A_{i}^{\mathrm{T}} P+P A_{i}=\left(\begin{array}{ccc}
0 & 0 & \alpha_{i} \\
0 & 0 & \beta_{i} \\
\alpha_{i} & \beta_{i} & \gamma_{i}
\end{array}\right), \quad \gamma_{i} \leqslant 0, \quad \forall i \in\{1, \cdots, 8\} .
$$

The spectrum of $A_{i}^{\mathrm{T}} P+P A_{i}, \operatorname{sp}\left(A_{i}^{\mathrm{T}} P+P A_{i}\right)=\left\{0, \frac{1}{2}\left(\gamma_{i} \pm \sqrt{4 \alpha_{i}^{2}+4 \beta_{i}^{2}+\gamma_{i}^{2}}\right)\right\}$ is a subset of nonpositive numbers if and only if $\alpha_{i}=\beta_{i}=0$. In particular, we have $M=\bigcap_{i=1}^{8} M_{i}=$ $M_{i}=\left\{x \in \mathbb{R}^{3} \mid x_{3}=0\right\}$. When $P$ and the gain matrices $L_{i}$ are chosen in such a way that Assumption 1.2 holds, we have

$$
A_{i}=\frac{1}{L}\left(\begin{array}{ccc}
0 & 0 & \left(u_{2}-u_{1}\right) \mu_{1}-\left(u_{3}-u_{2}\right) \mu_{3} \\
0 & 0 & \left(u_{3}-u_{2}\right) \mu_{2}-\left(u_{2}-u_{1}\right) \mu_{3} \\
u_{1}-u_{2} & u_{2}-u_{3} & -R_{i}
\end{array}\right), \quad R_{i}>0, \quad \forall i \in\{1, \ldots, 8\} .
$$

with $\mu_{1}=\frac{p_{22} p_{33}}{\operatorname{det} P}, \mu_{2}=\frac{p_{11} p_{33}}{\operatorname{det} P}, \mu_{3}=\frac{p_{12} p_{33}}{\operatorname{det} P}$.

We want to know under which condition solutions to (4.1) converge to zero. All results from Section 4 leading to convergence to zero being dwell-time based, we may wonder which type of dwell-time hypothesis are satisfied by the multi-cell chopper. Unfortunately, the different modes of the three-cell converter do not admit any dwell-time, only the switches do have one. In other words, one may switch from mode $A_{i}$ to mode $A_{j}(i \neq j)$ in an as small time as he wants, but has to wait a positive minimum time between two switches of the same the switch. We thus consider the following assumption. 
Assumption 4.1 (Switch dwell-time). The time elapsed between two commutations of the same switch has a positive inferior limit.

Notice that Assumption 4.1 implies that there exists at least one mode which satisfies the weak dwell-time Assumption 2.7, but it turns out that its does not imply stronger assumptions on modes.

One may naturally wonder if Assumption 4.1 implies the convergence to zero of the solution to system (4.1). The answer is negative as we shall in the next section.

Notice moreover that although $\operatorname{ker} \sum_{i=1}^{8} \frac{1}{8} A_{i}=M$ (which shows that the three-cell converter satisfies the hypothesis of Proposition 3.19), the counter example given by Proposition 3.19 is no more valid since the dwell-time on switches has not been taken into account.

\subsection{The $\omega$-limit set of a trajectory of the 3-cell converter observer is not necessarily a singleton}

In the present section, we construct a trajectory of the 3-cell converter observer (4.1) whose $\omega$-limit set is not a singleton. Moreover, we will see that the trajectory can be constructed in such a way that 3.1 Assumptions 2.7, 3.1, 3.7 and 4.1 are satisfied.

First of all, let us rewrite system (4.1) in a simpler way. Notice that up to the change of coordinates $x \rightarrow P^{1 / 2}$ and the time reparametrization $t \mapsto L t$ (two transformation that do not altered the topological structure of $\omega$-limit sets), we may assume that all the $A_{i}$ 's have the form

$$
A_{i}=\left(\begin{array}{ccc}
0 & 0 & a_{1}^{i} \\
0 & 0 & a_{2}^{i} \\
-a_{1}^{i} & -a_{2}^{i} & -a_{3}^{i}
\end{array}\right), \quad\left(a_{1}^{i}, a_{2}^{i}\right) \in\{0, \pm 1\}^{2} \backslash\{ \pm(1,1)\}, \quad a_{3}^{i}>0 .
$$

We now rewrite the system (4.1) using spherical coordinates $z=(r, \theta, \varphi)$ defined by:

$$
\begin{aligned}
& x_{1}=r \cos \theta \cos \varphi \\
& x_{2}=r \sin \theta \cos \varphi \\
& x_{3}=r \sin \varphi .
\end{aligned}
$$

It is easy to check that:

$$
\begin{aligned}
\dot{r} & =-a_{3}^{i} r \sin ^{2} \varphi \\
\dot{\theta} & =\left(a_{2}^{i} \cos \theta-a_{1}^{i} \sin \theta\right) \tan \varphi \\
\dot{\varphi} & =-a_{3}^{i} \cos \varphi \sin \varphi-\left(a_{1}^{i} \cos \theta+a_{2}^{i} \sin \theta\right)
\end{aligned}
$$

which shows in particular that $\dot{r}(t)=o(\dot{\theta}(t))$ as $t$ approaches infinity since $\varphi(t)$ goes to zero as $\mathrm{t}$ goes to infinity. This means that, approaching infinity, a trajectory of system (4.1) looses less in norm than it can win in angular position $\theta$, which encourages us to believe that we can build a trajectory of system (4.1) whose $\omega$-limit set is not a singleton.

Before beginning with the construction of the trajectory, let us fix the set $\mathcal{K}$ in which the trajectory will lie and some notation. We set:

- $\mathcal{K}=\left[0, r_{0}\right] \times\left[\theta_{0}, \theta_{f}\right] \times\left[0, \varepsilon_{0}\right] \subset\{(r, \theta, \varphi) \mid \cos \theta>\sin \theta>0\}$

- $f(i, z)=-a_{1}^{i} \cos \theta-a_{2}^{i} \sin \theta, \quad g(i, z)=a_{2}^{i} \cos \theta-a_{1}^{i} \sin \theta$

- $\alpha_{1}^{i}=\min _{z \in \mathcal{K}}\{f(i, z)\}, \quad \alpha_{2}^{i}=\max _{z \in \mathcal{K}}\{f(i, z)\}$ 
- $\alpha=\min _{\{z \in \mathcal{K}, i \neq 7,8\}}\{|\dot{\varphi}(i, z)|,|g(i, z)|\}, \quad \beta=\max _{\{z \in \mathcal{K}, i \neq 7,8\}}\{|\dot{\varphi}(i, z)|,|g(i, z)|\}$

- If $\left(v_{n}\right)_{n \in \mathbb{N}}$ is a sequence we denote by $\left(S(v)_{n}\right)_{n \in \mathbb{N}}$ the sequence of its partial sums, i.e., $S(v)_{n}=\sum_{k=0}^{n} v_{k}$.

Fix $\varepsilon_{0}>0$ so small that we have $\alpha>0, \beta>0$ and $\alpha_{j}^{i} \neq 0$ for every $j \in\{1,2\}$ and every $i \neq 7,8$.

\section{$1^{\text {st }}$ step: Construction of the switching trajectory lying in $\mathcal{K}$}

We will construct here a trajectory $x(\cdot)$ of system (4.1) such that $x(t) \in \mathcal{K}$ for all positive time $t$. In order to choose an order for the concatenation of the modes, let us make a sign table of velocities in $\mathcal{K}$.

\begin{tabular}{|c|c|c|c|c|c|c|c|c|}
\hline signs in $\mathcal{K}$ & 1 & 2 & 3 & 4 & 5 & 6 & 7 & 8 \\
\hline$\dot{\theta}$ & + & - & - & + & + & - & 0 & 0 \\
\hline$\dot{\varphi}$ & + & - & + & - & + & - & 0 & 0 \\
\hline
\end{tabular}

According to the sign table (4.5), one sees that the forward motions in $\theta$ are the flows $e^{t A_{1}}$, $e^{t A_{4}}, e^{t A_{5}}$ and the backward motions in $\theta$ the flows $e^{t A_{2}}, e^{t A_{3}}, e^{t A_{6}}$. For simplicity, we will only use the modes 2, 3, 4 and 5 for the construction of the trajectory. These modes correspond to the matrices

$A_{2}=\left(\begin{array}{ccc}0 & 0 & 1 \\ 0 & 0 & -1 \\ -1 & 1 & -a_{3}^{2}\end{array}\right), \quad A_{3}=\left(\begin{array}{ccc}0 & 0 & 0 \\ 0 & 0 & -1 \\ 0 & 1 & -a_{3}^{3}\end{array}\right), \quad A_{4}=\left(\begin{array}{ccc}0 & 0 & 0 \\ 0 & 0 & 1 \\ 0 & -1 & -a_{3}^{4}\end{array}\right), \quad A_{5}=\left(\begin{array}{ccc}0 & 0 & -1 \\ 0 & 0 & 1 \\ 1 & -1 & -a_{3}^{5}\end{array}\right)$,

From (4.4), one infers that $-a_{3}^{i} \varphi+\alpha_{1}^{i} \leqslant \dot{\varphi} \leqslant-a_{3}^{i} \varphi+\alpha_{2}^{i}$. Hence, as long as $\theta(t)$ stays in $\left[\theta_{0}, \theta_{f}\right]$, we have

$$
y_{1}^{i}(t) \leqslant \varphi(t) \leqslant y_{2}^{i}(t)
$$

where $y_{j}^{i}\left(\cdot, t_{0}\right)$ is the solution of the Cauchy problem $\dot{y}=-a_{3}^{i} y+\alpha_{j}^{i}, y\left(t_{0}\right)=\varphi\left(t_{0}\right)$, i.e.,

$$
y_{j}^{i}\left(t, t_{0}\right)=e^{-a_{3}^{i} t} \varphi\left(t_{0}\right)+\frac{\alpha_{j}^{i}}{a_{3}^{i}}\left(1-e^{-a_{3}^{i}\left(t-t_{0}\right)}\right) .
$$

\section{Construction of the $\varepsilon_{0}$-forward motion from $\theta_{0}$ to $\theta_{f}$}

Denote by $\Phi$ the inverse map of diffeomorphism $(r, \theta, \varphi) \mapsto(r \cos \theta \cos \varphi, r \sin \theta \cos \varphi, r \sin \varphi)$. For every $i \in\{1, \cdots, 8\}$, according to (4.2)-(4.4), the projection onto $(\theta, \varphi)$ of the pushforward by $\Phi$ of the field $A_{i}$ is a well-defined nonlinear autonomous vector field in the variables $(\theta, \varphi)$ which we denote by $\bar{A}_{i}$. Let $e^{t \bar{A}_{i}}$ denote the flow of $\bar{A}_{i}$ and $\pi$ be the projection $z \mapsto(\theta, \varphi)$.

Set $x_{0}=\left(r_{0}, \theta_{0}, 0\right)$ to be the initial condition and $t_{0}=0$ the initial time. We begin with following the mode $A_{5}$. Define $\tau_{1}=\inf \left\{t>0 \mid e^{t A_{5}}\left(x_{0}\right) \in \partial \mathcal{K}\right\}$. Necessarily, $\tau_{1}<+\infty$. If not, we would have $\theta(t)<\theta_{f}$ for all positive time, which, according to (4.7) and (4.6), would imply that

$$
\varphi(t) \geqslant y_{1}^{5}(t) \underset{t \rightarrow+\infty}{\rightarrow} \frac{\alpha_{1}^{5}}{a_{3}^{5}}>0,
$$

which is impossible since $\varphi(t)$ tends to zero as $t$ goes to infinity.

First case: $\boldsymbol{\theta}\left(\boldsymbol{\tau}_{\mathbf{1}}\right)=\boldsymbol{\theta}_{\boldsymbol{f}}$. For the same reason as $\tau_{1}$, $\inf \left\{t>0 \mid e^{-t \bar{A}_{4}}\left(\theta_{f}, 0\right) \in \partial \pi \circ\right.$ $\Phi(\mathcal{K})\}$, is a positive real number which implies that there must exists $\delta_{1}, \delta_{2}>0$ such that $\pi\left(\Phi\left(e^{\delta_{1} A_{5}}\left(x_{0}\right)\right)\right)=e^{-\delta_{2} \bar{A}_{4}}\left(\theta_{f}, 0\right)$, i.e., such that $\pi \circ \Phi\left(e^{\delta_{2} A_{4}} \circ e^{\delta_{1} A_{5}}\left(x_{0}\right)\right)=\left(\theta_{f}, 0\right)$.

Second case: $\boldsymbol{\theta}\left(\boldsymbol{\tau}_{\mathbf{1}}\right)<\boldsymbol{\theta}_{\boldsymbol{f}}$. Then, by construction, $\varphi\left(\tau_{1}\right)=\varepsilon_{0}$. In such a case, we define $\tau_{2}$ by $\tau_{2}=\inf \left\{t>0 \mid e^{t A_{4}} \circ e^{\tau_{1} A_{5}}\left(x_{0}\right) \in \partial \mathcal{K}\right\}$, which belongs to $\mathbb{R}_{+} \backslash\{0\}$ for the same 
reason as $\tau_{1}$. As in the first case, if $\theta\left(\tau_{2}\right)=\theta_{f}$, there must exists $\delta_{1}, \delta_{2}>0$ such that $\pi\left(\Phi\left(e^{\delta_{1} A_{5}}\left(x_{0}\right)\right)\right)=e^{-\delta_{2} \bar{A}_{4}}\left(\theta_{f}, 0\right)$. Indeed, the nonexistence of such $\delta_{1}, \delta_{2}$ would imply that

$$
\begin{aligned}
& \left\{e^{-\delta_{2} \bar{A}_{4}}\left(\theta_{f}, 0\right) \mid t>0\right\} \cap\left\{\pi \circ \Phi\left(e^{t A_{4}} \circ e^{\tau_{1} A_{5}}\left(x_{0}\right)\right) \mid t>0\right\}= \\
& =\left\{e^{-\delta_{2} \bar{A}_{4}}\left(\theta_{f}, 0\right) \mid t>0\right\} \cap\left\{e^{t \bar{A}_{4}}\left(\pi \circ \Phi\left(e^{\tau_{1} A_{5}}\left(x_{0}\right)\right)\right) \mid t>0\right\} \neq \emptyset,
\end{aligned}
$$

which contradicts the uniqueness theorem for solutions to ODEs. If $\theta\left(\tau_{2}\right)<\theta_{f}$, we set $\delta_{1}=\tau_{1}$. Then, define $\tau_{3}$ by $\tau_{3}=\inf \left\{t>0 \mid e^{t A_{5}} \circ e^{\tau_{2} A_{4}} \circ e^{\delta_{1} A_{5}}\left(x_{0}\right) \in \partial \mathcal{K}\right\}$, and go back, as with $\tau_{1}$ to the distinction between first and second case and so on. Step by step, we iteratively construct a sequence $\left(t_{n}\right)_{n \in \mathbb{N}}=\left(S(\delta)_{n}\right)_{n \in \mathbb{N}}$ of switching times such that $\theta\left(t_{n}\right) \in\left[\theta_{0}, \theta_{f}\right]$ and $\varphi\left(t_{n}\right) \in\left[0, \varepsilon_{0}\right]$ for all $n$. We next show that this process must stop. That is, we reach $\theta_{f}$ after finitely many commutations. To show this let us first evaluate the time elapsed between two consecutive commutations.

Evaluation of $\delta_{n+1}-\delta_{n}$

According to the mean value theorem, for every $\xi \in\left(t_{n}, t_{n+1}\right]$, there exists $c \in\left(t_{n}, \xi\right)$ such that $\left|\varphi(\xi)-\varphi\left(t_{n}\right)\right|=|\dot{\varphi}(c)|\left(\xi-t_{n}\right)$. Consequently, according to the definition of $\alpha$ and $\beta$,

$$
0<\alpha \leqslant \frac{\left|\varphi(\xi)-\varphi\left(t_{n}\right)\right|}{\xi-t_{n}} \leqslant \beta, \quad \forall \xi \in\left(t_{n}, t_{n+1}\right] .
$$

Suppose to reach a contradiction that $\theta\left(t_{n}\right)<\theta_{f}$ for infinitely many $n$. In this case, we have $\left|\varphi\left(t_{n+1}\right)-\varphi\left(t_{n}\right)\right|=\varepsilon_{0}$. At this point, integrating (4.3) we can evaluate the covered distance in $\theta$. According to (4.8) and because $\dot{\theta}(t), \varphi(t) \geqslant 0$, we get

$$
\begin{aligned}
\left|\theta\left(t_{2 n+1}\right)-\theta_{0}\right| & =\sum_{k=0}^{2 n} \int_{t_{k}}^{t_{k+1}} \dot{\theta}(\xi) d \xi \geqslant \sum_{k=0}^{2 n} \int_{t_{k}}^{t_{k+1}} \alpha \varphi(\xi) d \xi \\
& \geqslant \sum_{k=0}^{n} \int_{t_{2 k}}^{t_{2 k+1}} \alpha\left|\varphi(\xi)-\varphi\left(t_{2 k}\right)\right| d \xi \quad\left(t_{2 k}=0\right) \\
& \geqslant \sum_{k=0}^{n} \int_{t_{2 k}}^{t_{2 k+1}} \alpha^{2}\left(\xi-t_{k}\right) d \xi=\sum_{k=0}^{n} \frac{\alpha^{2}}{2}\left(t_{2 k+1}-t_{2 k}\right)^{2} \\
& \geqslant n \frac{\alpha^{2} \varepsilon_{0}^{2}}{2 \beta^{2}} \underset{n \rightarrow+\infty}{\rightarrow}+\infty,
\end{aligned}
$$

which contradicts the fact that $\theta\left(t_{n}\right)<\theta_{f}$ for infinitely many $n$. Let $N_{0}=N\left(\varepsilon_{0}\right)$ denote the number of switching times during the constructed $\varepsilon_{0}$-forward motion. Notice that $N_{0}$ is even if the initial time $\left(t_{0}=0\right)$ is counted as the first switching time. Once $\theta_{f}$ has been reached, in the same manner as the $\varepsilon_{0}$-forward motion was constructed, we use the flows $e^{t A_{2}}$ and $e^{t A_{3}}$ in order to construct an $\varepsilon_{1}$-backward motion (with $\varepsilon_{1} \leqslant \varepsilon_{0}$ ) to go back to $\theta_{0}$. Step by step, we iteratively construct a switching trajectory of system (4.1) which is a concatenation of $\varepsilon_{n}$-motions where $\left(\varepsilon_{n}\right)_{n \in \mathbb{N}}$ is a chosen sequence of positive numbers decreasing to zero. Let $N_{n}$ denote the number of commutations during an $\varepsilon_{n}$-motion. By construction, the trajectory $t \mapsto x(t)$ satisfies for every $n \in \mathbb{N}$ :

$$
\begin{array}{lr}
\varphi\left(t_{2 k}\right)=0, & \forall k \in \mathbb{N}, \\
\left|\varphi\left(t_{k+1}\right)-\varphi\left(t_{k}\right)\right|=\varepsilon_{n}, & \forall k \in\left\{S(N)_{n-1}+1, S(N)_{n}-2\right\}, \\
\left|\varphi\left(t_{S(N)_{n}}\right)-\varphi\left(t_{S(N)_{n}-1}\right)\right|=\gamma_{n}, & 0<\gamma_{n} \leqslant \varepsilon_{n}, \\
\left|\theta\left(t_{S(N)_{n+1}}\right)-\theta\left(t_{S(N)_{n}}\right)\right|=\theta_{f}-\theta_{0}, &
\end{array}
$$


where $\gamma_{n}$ corresponds to the value of $\varphi$ at the last switching time of the $\varepsilon_{n}$-motion.

$2^{\text {nd }}$ step: evaluation of the number of switching times during an $\varepsilon_{n}$-motion

One easily repeats on the time interval of an $\varepsilon_{n}$-motion a computation similar to (4.9) to conclude that

$$
\theta_{f}-\theta_{0}=\theta\left(t_{S(N)_{n}+1}\right)-\theta\left(t_{S(N)_{n}}\right) \geqslant\left(\left(N_{n} / 2-1\right) \varepsilon_{n}^{2}+\gamma_{n}^{2}\right) \frac{\alpha^{2}}{2 \beta^{2}}
$$

from which it follows that

$$
N_{n} \leqslant 4 \beta^{2}\left(\theta_{f}-\theta_{0}\right) /\left(\alpha^{2} \varepsilon_{n}^{2}\right)+2\left(\varepsilon_{n}^{2}-\gamma_{n}^{2}\right) / \varepsilon_{n}^{2} \leqslant C / \varepsilon_{n}^{2},
$$

with $C=\left(4 \beta^{2}\left(\theta_{f}-\theta_{0}\right)+2 \alpha^{2} \varepsilon_{0}^{2}\right) / \varepsilon_{n}^{2}$.

\section{$3^{\text {rd }}$ step: evaluation of the loss in norm}

The last thing we have to do is to evaluate the loss in norm during along the whole trajectory. Let us first estimate the loss in norm during an $\varepsilon_{n}$-motion. Denote by $\left[b_{0}^{n}, b_{N_{n}}^{n}\right]$ the time intervals corresponding to this motion. Set $a=\max \left\{a_{3}^{1}, \ldots, a_{3}^{8}\right\}$. According to (4.2), (4.8) and (4.10), one infers that

$$
\begin{aligned}
r\left(b_{0}^{n}\right)-r\left(b_{N_{n}}^{n}\right) & =\int_{b_{0}^{n}}^{b_{N_{n}}^{n}}|\dot{r}(\xi)| d \xi \leqslant \int_{b_{0}^{n}}^{b_{N_{n}}^{n}} a r_{0} \varphi^{2}(\xi) d \xi \\
& \leqslant a r_{0} \varepsilon_{n}^{2} \int_{b_{0}^{n}}^{b_{N_{n}}^{n}} d \xi=a r_{0} \varepsilon_{n}^{2} \sum_{k=1}^{N_{n}}\left|b_{k}^{n}-b_{k-1}^{n}\right| d \xi \\
& \leqslant a r_{0} \varepsilon_{n}^{2} \sum_{k=1}^{N_{n}} \alpha^{-1}\left|\varphi\left(b_{k}^{n}\right)-\varphi\left(b_{k-1}^{n}\right)\right| d \xi \\
& \leqslant N_{n} a r_{0} \alpha^{-1} \varepsilon_{n}^{3} \leqslant \widetilde{C} \varepsilon_{n},
\end{aligned}
$$

where $\widetilde{C}=a r_{0} C / \alpha$. Consequently, the loss in norm up to time $b_{N_{n}}^{n}$ is

$$
r_{0}-r\left(b_{N_{n}}^{n}\right)=\sum_{k=0}^{n}\left|r\left(b_{0}^{k}\right)-r\left(b_{N_{k}}^{k}\right)\right| \leqslant \widetilde{C} \sum_{k=0}^{n} \varepsilon_{n},
$$

And, passing to the limit as $n$ tends to infinity, we get

$$
r_{0}-\lim _{t \rightarrow+\infty} r(t)=r_{0}-\lim _{n \rightarrow+\infty} r\left(b_{N_{n}}^{n}\right) \leqslant \widetilde{C} \sum_{k=0}^{+\infty} \varepsilon_{n},
$$

which can be chosen to be strictly less than $r_{0}$. In such a case, $\lim _{t \rightarrow+\infty} r(t)=r_{f} \neq 0$ and by construction, $\omega(r(t), \theta(t), \varphi(t))=\left\{r_{f}\right\} \times\left[\theta_{0}, \theta_{f}\right] \times\{0\}$ which is not a singleton.

\section{$4^{\text {th }}$ and last step: ensuring Assumption 4.1}

The constructed trajectory violates Assumption 4.1 (switch dwell-time). We show here that we can slightly modify this trajectory so that it will respect the dwell-time on switches. Notice that during a forward motion the commutation from mode 5 to mode 4 involves only the use of switch $u_{1}$. Thus, at every switching time $t_{2 k}(k \in \mathbb{N})$, instead of switching back to mode mode 5 we can switch to mode 8 using the switch $u_{3}$. Since $x\left(t_{2 k}\right) \in \operatorname{ker} A_{8}=\left\{x_{3}=0\right\}$, we have $e^{\tau A_{8}}\left(x\left(t_{2 k}\right)\right)=x\left(t_{2 k}\right)$ for every positive $\tau$. Choosing $\tau$ greater than the switch dwell-time shows that any forward $\varepsilon_{2 k}$-motion can be done respecting Assumption 4.1. The 
reader can easily check that the same can also be done for every backward $\varepsilon_{2 k+1}$-motion. Consequently, we can assume that the constructed trajectory satisfies Assumption 4.1. Even more, by complicating somewhat the way we construct the trajectory, we can involve each matrix of the family $\left\{A_{1}, \ldots, A_{8}\right\}$ in such a way that the assumption of persistent excitation (Assumption 3.7) is satisfied.

Final remark. It is easy to see that one can construct a trajectories of system (4.1) whose $\omega$-limit sets does not reduce to a singleton which satisfy both the weak dwell-time and the switch dwell-time assumptions. Such trajectories can be can be constructed as soon as the number cells in the converter is greater or equal to three. When the number of cells is smaller or equal to two, Proposition 3.6 shows that the weak-time assumption is sufficient to insure the convergence of the trajectories to zero.

\section{References}

[1] A. A. Agrachev and Y. L. Sachkov. Control theory from the geometric viewpoint, volume 87 of Encyclopaedia of Mathematical Sciences. Springer-Verlag, Berlin, 2004. Control Theory and Optimization, II.

[2] A. Bacciotti and L. Mazzi. An invariance principle for nonlinear switched systems. Systems Control Lett., 54(11):1109-1119, 2005.

[3] K. Benmansour, J. de Leon, and M. Djemaï. Adaptive observer for multi-cell chopper. In Second International Symposium on Communications, Control and Signal Processing ISCCSP, Marrakech, Maroc, 2006.

[4] K. Benmansour, M. Djemaï, M. Tadjine, and M. Boucherit. On sliding mode observer for hybrid three cells converter : Experimental results. International Workshop on Variable Structure Systems, 2008. VSS '08., pages 373-377, June 2008.

[5] R. Bensaid and M. Fadel. Floating voltages estimation in three-cell converters using a discrete-time kalman filter. Power Electronics Specialists Conference, 2001. PESC. 2001 IEEE 32nd Annual, 1:327-332 vol. 1, 2001.

[6] M. S. Branicky. Multiple Lyapunov functions and other analysis tools for switched and hybrid systems. IEEE Trans. Automat. Control, 43(4):475-482, 1998. Hybrid control systems.

[7] H. Brezis. Analyse fonctionnelle. Collection Mathématiques Appliquées pour la Maîtrise. Masson, Paris, 1983.

[8] V. Chellaboina, S. P. Bhat, and W. M. Haddad. An invariance principle for nonlinear hybrid and impulsive dynamical systems. Nonlinear Anal., 53(3-4):527-550, 2003.

[9] F. Colonius and W. Kliemann. The dynamics of control. Systems \& Control: Foundations \& Applications. Birkhäuser Boston Inc., Boston, MA, 2000. With an appendix by Lars Grüne.

[10] J. Daafouz, P. Riedinger, and C. Iung. Stability analysis and control synthesis for switched systems: a switched Lyapunov function approach. IEEE Trans. Automat. Control, 47(11):1883-1887, 2002. 
[11] R. A. Decarlo, M. S. Branicky, S. Pettersson, and B. Lennartson. Perspectives and results on the stability and stabilizability of hybrid systems. In Proceedings of the IEEE, volume 88, pages 1069-1082, 2000.

[12] R. Goebel, R. G. Sanfelice, and A. R. Teel. Invariance principles for switching systems via hybrid systems techniques. Systems Control Lett., 57(12):980-986, 2008.

[13] J. P. Hespanha. Uniform stability of switched linear systems: extensions of LaSalle's invariance principle. IEEE Trans. Automat. Control, 49(4):470-482, 2004.

[14] V. Jurdjevic. Geometric control theory, volume 52 of Cambridge Studies in Advanced Mathematics. Cambridge University Press, Cambridge, 1997.

[15] V. Jurdjevic and I. Kupka. Control systems on semisimple Lie groups and their homogeneous spaces. Ann. Inst. Fourier (Grenoble), 31(4):vi, 151-179, 1981.

[16] P. E. Kloeden. Nonautonomous attractors of switching systems. Dyn. Syst., 21(2):209$230,2006$.

[17] D. Liberzon. Switching in systems and control. Systems \& Control: Foundations \& Applications. Birkhäuser Boston Inc., Boston, MA, 2003.

[18] D. Liberzon, J. P. Hespanha, and A. S. Morse. Stability of switched systems: a Liealgebraic condition. Systems Control Lett., 37(3):117-122, 1999.

[19] J. Lygeros, K. H. Johansson, S. N. Simić, J. Zhang, and S. S. Sastry. Dynamical properties of hybrid automata. IEEE Trans. Automat. Control, 48(1):2-17, 2003.

[20] J. L. Mancilla-Aguilar and R. A. García. An extension of LaSalle's invariance principle for switched systems. Systems Control Lett., 55(5):376-384, 2006.

[21] S. Nelson and M. Neumann. Generalizations of the projection method with applications to SOR theory for Hermitian positive semidefinite linear systems. Numer. Math., 51(2):123$141,1987$.

[22] W. Rudin. Real and complex analysis. McGraw-Hill Book Co., New York, third edition, 1987.

[23] R. G. Sanfelice, R. Goebel, and A. R. Teel. Invariance principles for hybrid systems with connections to detectability and asymptotic stability. IEEE Trans. Automat. Control, 52(12):2282-2297, 2007.

[24] R. Shorten, F. Wirth, O. Mason, K. Wulff, and C. King. Stability criteria for switched and hybrid systems. SIAM Rev., 49(4):545-592, 2007.

[25] B. Sz.-Nagy and C. Foiaş. Harmonic analysis of operators on Hilbert space. Translated from the French and revised. North-Holland Publishing Co., Amsterdam, 1970. 